Mon. Not. R. Astron. Soc. 000, 000-000 (0000) Printed 1 November $2018 \quad$ (MN LATEX style file v2.2)

\title{
CPD-64 2731: a massive spun-up and rejuvenated high-velocity runaway star
}

\author{
V. V. Gvaramadze, ${ }^{1,2,3 \star}$ O. V. Maryeva, ${ }^{4,1}$ A. Y. Kniazev, ${ }^{5,6,1}$ D. B. Alexashov, ${ }^{7,2}$ \\ N. Castro, ${ }^{8}$ N. Langer ${ }^{9}$ and I. Y. Katkov ${ }^{1}$ \\ ${ }^{1}$ Sternberg Astronomical Institute, Lomonosov Moscow State University, Universitetskij Pr. 13, Moscow 119992, Russia \\ ${ }^{2}$ Space Research Institute, Russian Academy of Sciences, Profsoyuznaya 84/32, 117997 Moscow, Russia \\ ${ }^{3}$ Isaac Newton Institute of Chile, Moscow Branch, Universitetskij Pr. 13, Moscow 119992, Russia \\ ${ }^{4}$ Astronomical Institute, Czech Academy of Sciences, Fričova 298, 25165 Ondřejov, Czech Republic \\ ${ }^{5}$ South African Astronomical Observatory, PO Box 9, 7935 Observatory, Cape Town, South Africa \\ ${ }^{6}$ Southern African Large Telescope Foundation, PO Box 9, 7935 Observatory, Cape Town, South Africa \\ ${ }^{7}$ Institute for Problems in Mechanics, prosp. Vernadskogo 101, block 1, Moscow 119526, Russia \\ ${ }^{8}$ Department of Astronomy, University of Michigan, 1085 S. University Avenue, Ann Arbor, MI 48109-1107, USA \\ ${ }^{9}$ Argelander-Institut für Astronomie, Auf dem Hügel 71, D-53121 Bonn, Germany
}

Accepted 2018 October 26. Received 2018 October 12; in original form 2018 September 14

\begin{abstract}
We report the results of our study of the high-velocity $\left(\approx 160 \mathrm{~km} \mathrm{~s}^{-1}\right)$ runaway $\mathrm{O}$ star $\mathrm{CPD}-64^{\circ} 2731$ and its associated horseshoe-shaped nebula discovered with the Widefield Infrared Survey Explorer. Spectroscopic observations with the Southern African Large Telescope and spectral analysis indicate that $\mathrm{CPD}-64^{\circ} 2731$ is a fast-rotating main-sequence O5.5 star with enhanced surface nitrogen abundance. We derive a projected rotational velocity of $\approx 300 \mathrm{~km} \mathrm{~s}^{-1}$ which is extremely high for this spectral type. Its kinematic age of $\approx 6 \mathrm{Myr}$, assuming it was born near the Galactic plane, exceeds its age derived from single star models by a factor of two. These properties suggest that CPD $-64^{\circ} 2731$ is a rejuvenated and spun-up binary product. The geometry of the nebula and the almost central location of the star within it argue against a pure bow shock interpretation for the nebula. Instead, we suggest that the binary interaction happened recently, thereby creating the nebula, with a cavity blown by the current fast stellar wind. This inference is supported by our results of $2 \mathrm{D}$ numerical hydrodynamic modelling.
\end{abstract}

Key words: blue stragglers - circumstellar matter - stars: individual: CPD-64 ${ }^{\circ} 2731$ - stars: massive - stars: rotation

\section{INTRODUCTION}

Wind parameters of (single) massive stars, such as the terminal wind velocity and mass-loss rate, undergo significant changes in the course of stellar evolution, which can lead to the formation of transient circumstellar nebulae around evolved massive stars. For example, the origin of circumstellar nebulae around late nitrogen-sequence Wolf-Rayet (WNL) stars could be attributed to the interaction between the fast wind of these stars and a slow dense material shed during preceding evolutionary stages (e.g. García-Segura, Mac Low \& Langer 1996a; García-Segura, Langer \& Mac Low 1996b; Brighenti \& D'Ercole 1997). The circumstellar nebulae are generally observed around field (i.e. runaway)

* E-mail: vgvaram@mx.iki.rssi.ru stars or around stars at the outskirts of their parent star clusters, where winds from other cluster members do not prevent their formation.

Observations show that the majority of massive stars are formed in binary and multiple systems (Mason et al. 2009; Sana \& Evans 2011; Chini et al. 2012). The mass loss from these systems is defined not only by the (variable) stellar wind of individual companion stars, but also by various binary interaction processes, such as mass transfer or merger. In both cases, the system can lose a significant fraction of its mass (up to several solar masses) which slowly spreads into the circumstellar environment (e.g. Petrovic, Langer \& van der Hucht 2005; Glebbeek et al. 2013). After the thermal adjustment on the Kelvin-Helmholtz time-scale, the mass gainer or merger product might become a source of fast wind, which will blow a bubble in the surrounding 


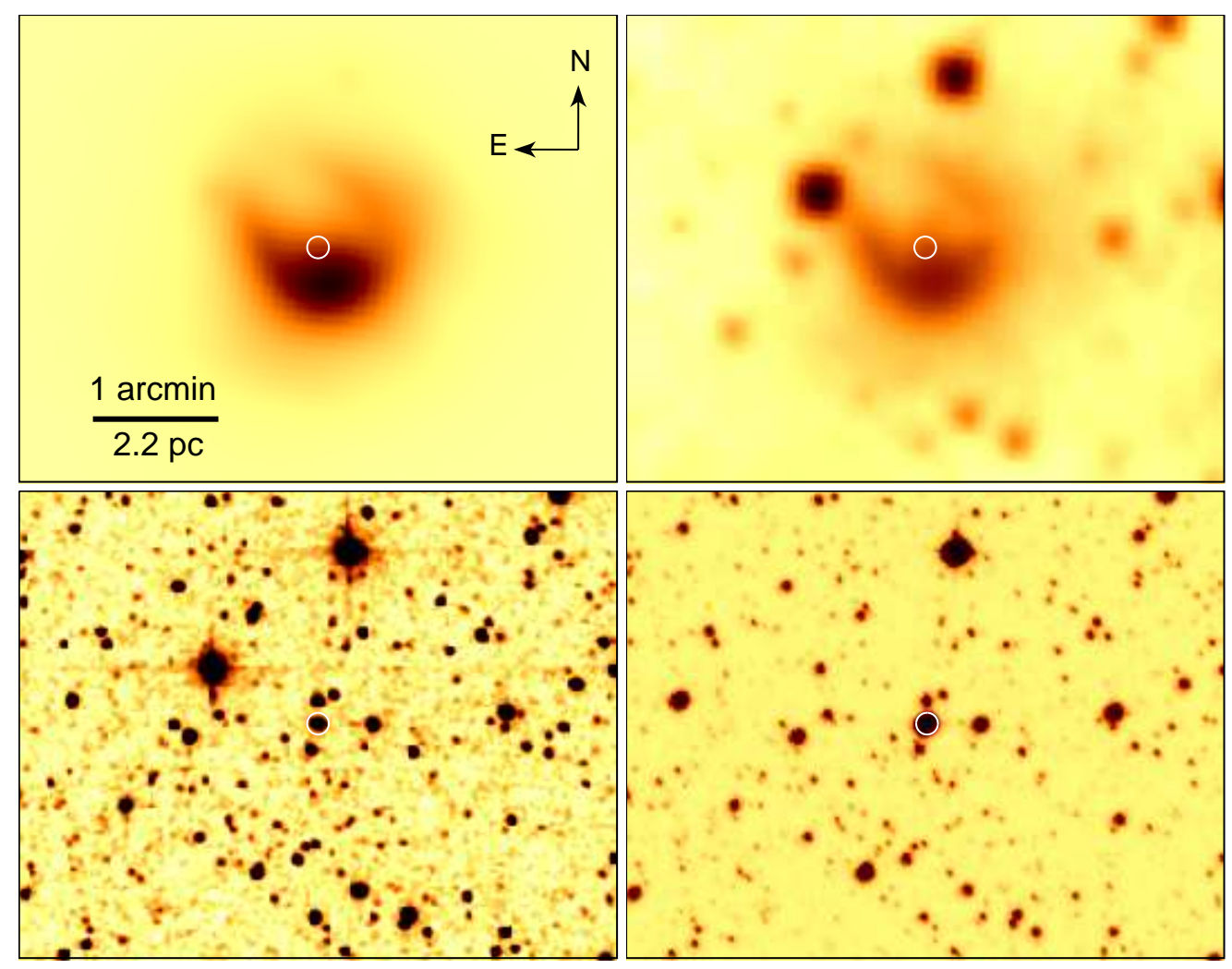

Figure 1. From left to right, and from top to bottom: WISE 22 and $12 \mu \mathrm{m}, 2 \mathrm{MASS} K_{\mathrm{s}}$-band and SHS H $\alpha$ images of the region containing $\mathrm{CPD}-64^{\circ} 2731$ (marked by a circle) and its surrounding infrared nebula. The orientation and the scale of the images are the same.

medium. This bubble would be surrounded by a shell (nebula) provided that its expansion is supersonic. The shell will not form if the binary interaction occurred within a larger hot bubble produced by winds of all massive stars of the parent star cluster. Thus, to produce a detectable nebula, the binary system should find itself far from the birth cluster well prior to the beginning of the mass transfer or merger event.

Observations also show that $\approx 70$ per cent of runaway $\mathrm{O}$ stars are binary systems (Chini et al. 2012). Moreover, about 40 per cent of $\mathrm{O}$ stars interact with a companion while they are still on the main sequence (Sana et al. 2012). This implies that many of the main-sequence $\mathrm{O}$ stars in the field could be the rejuvenated products of mass transfer or binary mergers (runaway blue stragglers), and therefore some of them could be surrounded by initially almost spherical nebulae, whose origin hardly can be explained within the framework of single star evolution. These nebulae, however, should be extremely rare because they rapidly transform into bow shocks when the forward edge of the wind bubble crosses the region occupied by the slow material lost during the binary interaction process and starts to interact with the unperturbed interstellar medium (ISM).

In this paper, we report the discovery of an almost complete mid-infrared shell around the high-velocity runaway main-sequence $\mathrm{O}$ star $\mathrm{CPD}-64^{\circ} 2731$. In Section2 we present images of the nebula and review observational data on the star. Section 3 describes our optical spectroscopic observations of $\mathrm{CPD}-64^{\circ} 2731$. The spectral analysis of the star is given in Section 4 In Section 5 we show that CPD-64 2731 is a high-velocity runaway star, while in Section 6] we argue that CPD-64 2731 is a spun-up and rejuvenated product of binary interaction (massive blue straggler). In Sections 7 and 8, we discuss possible explanations for the origin of the nebula and verify them by means of $2 \mathrm{D}$ numerical simulations, respectively. Concluding remarks are given in Section9 We summarize in Section 10

\section{HORSESHOE-SHAPED NEBULA AND ITS CENTRAL STAR}

The nebula, which is the subject of this paper, was discovered in the Circinus constellation during our search for mid-infrared nebulae in areas of the sky that were not covered by the major Spitzer Space Telescope surveys of massive star-forming regions (for motivation of the search see Gvaramadze, Kniazev \& Fabrika 2010). For this purpose, we used data from the all-sky survey carried out by the Wide-field Infrared Survey Explorer (WISE), which provides images at four wavelengths: 3.4, 4.6, 12 and $22 \mu \mathrm{m}$, with angular resolution of $6.1,6.4,6.5$ and 12.0 arcsec, respectively (Wright et al. 2010).

The search has led to the discovery of many dozens of previously unknown nebulae. Some of them were found around newly identified massive stars, such as luminous blue variables and blue supergiants (e.g. Gvaramadze et al. 2012a). Some others were interpreted as bow shocks pro- 
Table 1. Details of CPD $-64^{\circ} 2731$. The spectral type, SpT, is based on our spectroscopic observations and spectral analysis. The coordinates and the $J H K_{\mathrm{S}}$ photometry are from the 2MASS All-Sky Catalog of Point Sources (Cutri et al. 2003), the $B V$ photometry is from the AAVSO Photometric All-Sky Survey (Henden et al. 2016), and the [3.4] and [4.6] magnitudes are from the AllWISE Data Release (Cutri et al. 2014).

\begin{tabular}{lc}
\hline $\mathrm{SpT}$ & $\mathrm{O} 5.5 \mathrm{Vn}((\mathrm{f}))$ \\
$\alpha(\mathrm{J} 2000)$ & $14^{\mathrm{h}} 07^{\mathrm{m}} 07^{\mathrm{S}} .2$ \\
$\delta(\mathrm{J} 2000)$ & $-65^{\circ} 29^{\prime} 34^{\prime \prime} 4$ \\
$l$ & $310^{\circ} 6792$ \\
$b$ & -3.7659 \\
$B$ (mag) & $10.872 \pm 0.020$ \\
$V$ (mag) & $10.597 \pm 0.015$ \\
$J$ (mag) & $10.053 \pm 0.024$ \\
$H$ (mag) & $9.998 \pm 0.025$ \\
$K_{\mathrm{s}}(\mathrm{mag})$ & $9.990 \pm 0.023$ \\
{$[3.4](\mathrm{mag})$} & $9.926 \pm 0.024$ \\
{$[4.6](\mathrm{mag})$} & $9.965 \pm 0.022$ \\
\hline
\end{tabular}

duced by known or newly identified OB stars (e.g. Gvaramadze et al. 2011). As a by-product, we discovered three planetary nebulae (e.g. Gvaramadze \& Kniazev 2017), one of which, indicated in the SIMBAD data bast 1 as a possible planetary nebula "NAME PN Ra 7" (Ferrero et al. 2015), was found to be associated with a [WO1] star (Gvaramadze et al., in preparation). And one of the nebulae detected with WISE turns out to be quite unusual because it is associated with a high-velocity runaway main-sequence $\mathrm{O}$ star, but did not look like a genuine bow shock.

Figure 1 shows WISE 22 and $12 \mu \mathrm{m}$ images of this nebula. In both images it appears as a horseshoe-shaped shell with angular radius of $\approx 30$ arcsec and enhanced brightness along the southern rim. The shell is not visible at shorter WISE wavelengths, nor in the optical images from the Digitized Sky Survey II (McLean et al. 2000) and the SuperCOSMOS H-alpha Survey (SHS; Parker et al. 2005). The horseshoe appearance of the shell and its brightness asymmetry suggest that the star producing the shell is moving to the south or southwest, and that the geometry of the shell is affected by the ram pressure of the incoming ISM flow.

Using the SIMBAD data base, we identified the nebula with the Infrared Astronomical Satellite (IRAS) source IRAS14032-6515, and found within it a massive star, known as CPD- $64^{\circ} 2731$ or ALS 3198 (marked in Fig. 1 by a circle). $\mathrm{CPD}-64^{\circ} 2731$ was recognized as an OB star by Lynga (1969) and later on was classified as a fast-rotating O5 giant (O5 IIIn) star by Vijapurkar \& Drilling (1993). In Fig.1 we also show the $K_{\mathrm{s}}$-band and $\mathrm{H} \alpha$ images of the field containing the nebula and $\mathrm{CPD}-64^{\circ} 2731$ taken, respectively, from the Two Micron All Sky Survey (2MASS; Skrutskie et al. 2006) and the SHS. One can see that the star is slightly offset (by $\approx 5$ arcsec) from the geometric centre of the nebula towards its brighter side.

The details of $\mathrm{CPD}-64^{\circ} 2731$ are summarized in Table1

\footnotetext{
${ }^{1}$ http://simbad.harvard.edu/simbad/
}

\section{SPECTROSCOPIC OBSERVATIONS}

CPD-64을 2731 was observed with the Southern African Large Telescope (SALT) High Resolution Spectrograph (HRS; Barnes et al. 2008; Bramall et al. 2010, 2012; Crause et al. 2014) at Medium Resolution (MR) mode on 2018 February 1 to verify its spectral type, and re-observed on 2018 February 25 and July 18 (during twilight time) to search for possible radial velocity variability. All observations were carried out with the same single exposure of $1200 \mathrm{~s}$. The seeing during these observations was, respectively, $\approx 1.6,2.6$ and 1.6 arcsec.

The HRS is a dual beam, fibre-fed échelle spectrograph. In the MR mode it has a 2.23 arcsec diameter for both the object and sky fibres, providing a spectrum in the blue and red arms over the total spectral range of $\approx 3700-8900 \AA$ with resolution of $\mathrm{R} \sim 35000$. For our observations both blue and red arms CCD were read out by a single amplifier with a $1 \times 1$ binning. Three spectra of the ThAr arc-lamp and three spectral flats were obtained in this mode during a weekly set of HRS calibrations. Primary reduction of the spectra was performed with the SALT science pipeline (Crawford et al. 2010). The subsequent reduction steps were carried out using MIDAS HRS pipeline described in details in Kniazev, Gvaramadze \& Berdnikov (2016). A part of the spectrum obtained on 2018 February 1 is shown in Fig.2

\section{SPECTRAL ANALYSIS AND STELLAR PARAMETERS}

\subsection{Classification of $\mathrm{CPD}-64^{\circ} 2731$}

Figure 2 shows that the spectrum of CPD- $64^{\circ} 2731$ is dominated by broad absorption lines of $\mathrm{H}$, He I and He II, indicating that we deal with a fast-rotating O star (cf. Vijapurkar \& Drilling 1993). Except of the weak P Cygni profile of the He II $\lambda 4686$ line, the only emission lines detected are those of $\mathrm{N}$ III $\lambda \lambda 4634,4641-42$, meaning that $\mathrm{CPD}-64^{\circ} 2731$ is an $\mathrm{O}((\mathrm{f}))$ star (e.g. Sota et al. 2011). Using equivalent widths (EW) of the H I $\lambda 4471$ and He II $\lambda 4541$ lines of $0.39 \pm 0.01 \AA$ and $0.88 \pm 0.01 \AA$, respectively, one finds $\log W=\log (\mathrm{EW}(\mathrm{H}$ I 4471) $/ \mathrm{EW}($ He II 4541) $=-0.35 \pm 0.01$, which implies that CPD-64 2731 is an O5.5 star (Conti \& Alschuler 1971; see their table 3$)$. To determine the luminosity class, we use the criteria from Sota et al. (2014; see their table 2), according to which CPD $-64^{\circ} 2731$ is a dwarf star (luminosity class V). To this classification we add a suffix ' $n$ ', indicating that the projected rotational velocity of $\mathrm{CPD}-64^{\circ} 2731$ is $\approx 300 \mathrm{~km} \mathrm{~s}^{-1}$ (see Section 4.2), so that $\mathrm{CPD}-64^{\circ} 2731$ is an $\mathrm{O} 5.5 \mathrm{Vn}((\mathrm{f}))$ star.

\subsection{Spectral modelling}

To determine stellar parameters and chemical abundances of CPD $-64^{\circ} 2731$, we used the stellar atmosphere code CMFGEN (Hillier \& Miller 1998). This code solves radiative transfer equations for objects with extended spherically symmetric outflows using either the Sobolev approximation or the full comoving-frame solution of the radiative transfer equation. CMFGEN incorporates line blanketing, the effects of Auger ionization and clumping. Every model is defined by a hydrostatic stellar radius $R_{*}$, luminosity $L_{*}$, mass-loss rate 


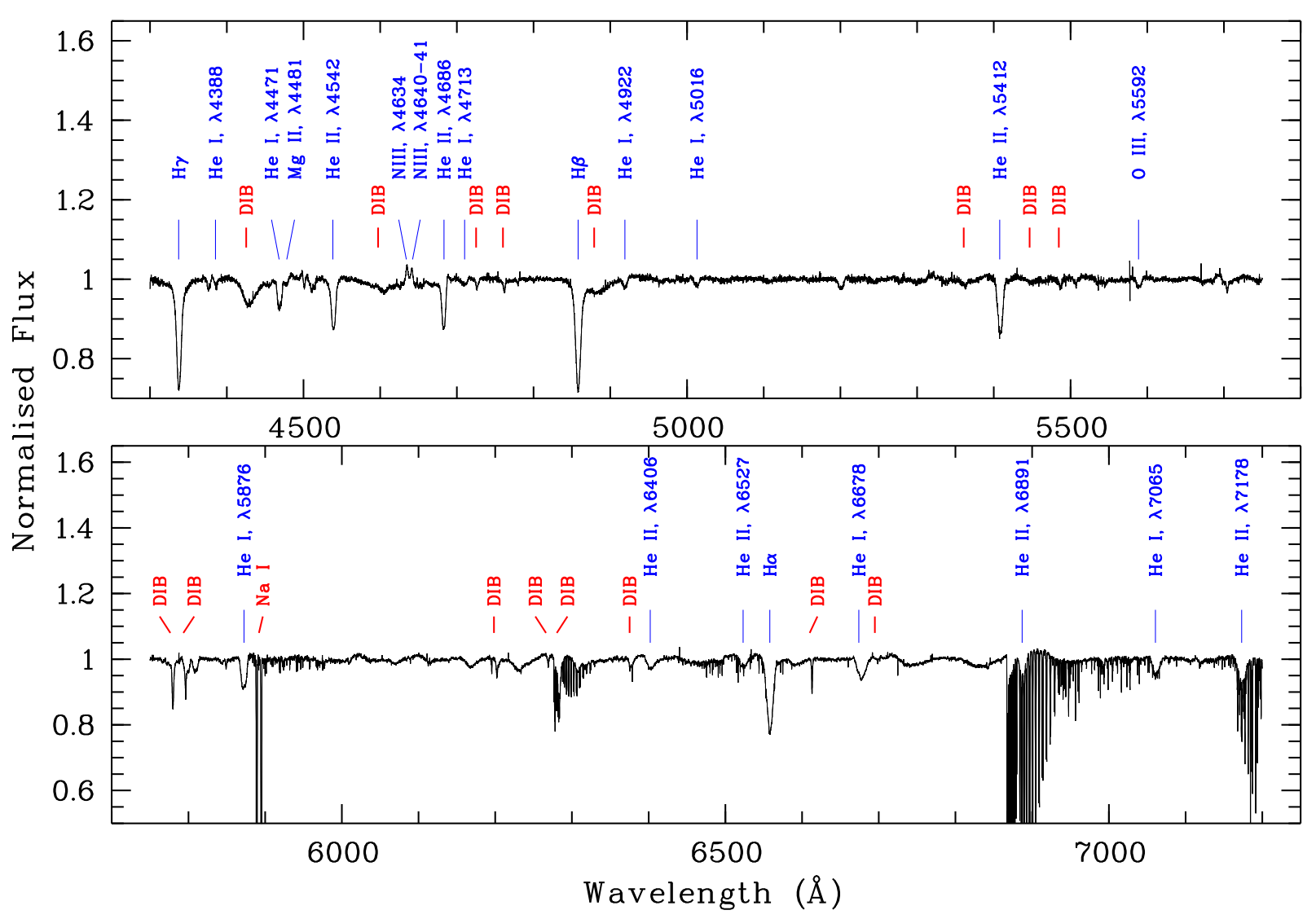

Figure 2. A part of the normalized spectrum of CPD-64을 2731 obtained with the SALT HRS on 2018 February 1 . The principal lines and most prominent diffuse interstellar bands (DIBs) are indicated.

$\dot{M}$, volume filling factor $f$, wind terminal velocity $v_{\infty}$, stellar mass $M_{*}$, and abundances $Z_{i}$ of chemical elements. Our modelling included the following elements: $\mathrm{H}, \mathrm{He}, \mathrm{C}, \mathrm{N}, \mathrm{O}$, $\mathrm{Si}, \mathrm{S}, \mathrm{P}$ and Fe. The best-fitting model is shown in Fig. 3.

For the initial photospheric density structure we adopted the TLUSTY hydrostatic model atmosphere of Hubeny \& Lanz (1995) and Lanz \& Hubeny (2003) for deep quasi-static layers connected to the wind with standard $\beta$ velocity law just above the sonic point. We chose $\beta=0.8$ as the default value for our calculation, which is a typical value of this parameter for $\mathrm{O}$ dwarf stars (e.g. Repolust, Puls \& Herrero 2004). In the spectrum of $\mathrm{CPD}-64^{\circ} 2731$ there are no lines with pronounced $\mathrm{P}$ Cyg profiles which can be used to measure $v_{\infty}$. We therefore assumed that $v_{\infty}=2.65 v_{\text {esc }}$ (Kudritzki \& Puls 2000), where $v_{\text {esc }}$ is the escape velocity. The mass-loss rate was estimated by fitting profiles of the $\mathrm{H} \alpha$ and He II $\lambda 4686$ lines.

The clumping was described by the volume filling factor $f=\bar{\rho} / \rho(r)$, where $\bar{\rho}$ is the homogeneous (unclumped) wind density and $\rho$ is the density in clumps, which are assumed to be optically thin (Hillier \& Miller 1999). The interclump medium is void. The filling factor depends on the radius as $f=f_{\infty}+\left(1-f_{\infty}\right) \exp \left(-v(r) / v_{\mathrm{cl}}\right)$, where $f_{\infty}$ describes the density contrast and $v_{\mathrm{cl}}$ is a velocity at which the clumping becomes important. We adopted $v_{\mathrm{cl}}=10 \mathrm{~km} \mathrm{~s}^{-1}$ and $f_{\infty}=$ 0.5 to simultaneously fit the $\mathrm{H} \alpha$ and He II $\lambda 4686$ lines.
To derive the effective temperature $T_{\text {eff }}$ (defined at the radius where the Rosseland optical depth equals $2 / 3$ ), we compared intensities of the He I and He II lines. The gravity was derived by fitting the wings of Balmer lines.

The hydrogen and helium abundances were derived by iterative adjustment of the He-to- $\mathrm{H}$ abundance ratio and other fundamental parameters of $\mathrm{CPD}-64^{\circ} 2731$ to reproduce the overall shape of all detected $\mathrm{H}$ and He lines. The nitrogen abundance was estimated by analysing the behaviour of all nitrogen lines in the spectrum. The oxygen and carbon abundances were derived by use of the $\mathrm{O}$ III $\lambda 5592$ and $\mathrm{C}$ IV $\lambda \lambda 5801,5812$ lines, respectively. Note that our best-fitting model shows the C III $\lambda \lambda 4647-50-52$ lines in emission, while they are absent in the observed spectrum. Modelling of these lines is a difficult task because of very complex dependence of their profiles on the main stellar parameters $\left(\log g, T_{\text {eff }}\right.$, $\dot{M})$ as well as on the inclusion of other ions in calculations, such as Fe IV, Fe V and S IV (Martins \& Hillier 2012). We suppose that the emergence of the C III $\lambda \lambda 4647-50-52$ lines is connected with the atomic data we used in our model. For the abundances of $\mathrm{Si}, \mathrm{S}, \mathrm{P}$ and Fe we adopted solar values.

In Table2, we compare the CNO abundances (by number) of $\mathrm{CPD}-64^{\circ} 2731$ with the cosmic abundance standard (CAS) in the solar neighbourhood (Nieva \& Przybilla 2012) and the initial CNO abundances adopted in the evolutionary models of Galactic O stars by Brott et al. (2011). One 

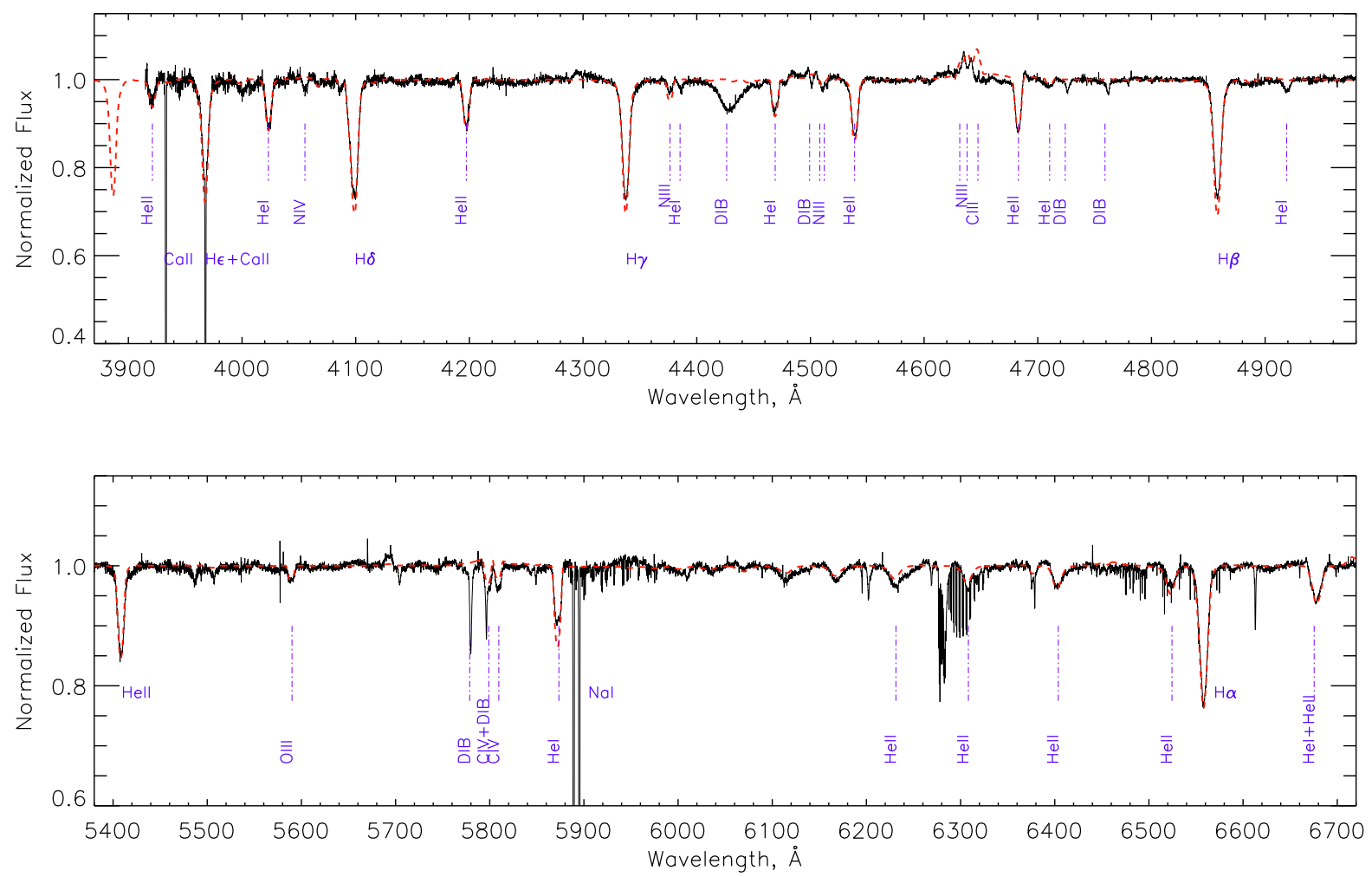

Figure 3. Normalized HRS spectrum of CPD-64 2731 taken on 2018 February 1 (black solid line), compared with the best-fitting CMFGEN model (red dashed line) with the parameters as given in Table 3

Table 2. Comparison of the $\mathrm{CNO}$ abundances (by number) in CPD $-64^{\circ} 2731$ with the cosmic abundance standard (CAS; Nieva \& Przybilla 2012) in the solar neighbourhood and initial abundances adopted in the evolutionary models by Brott et al. (2011).

\begin{tabular}{lccc}
\hline $\log (\mathrm{X} / \mathrm{H})+12$ & CPD-64 2731 & CAS & Brott et al. \\
\hline $\mathrm{C}$ & $8.30_{-0.30}^{+0.18}$ & $8.33 \pm 0.04$ & 8.13 \\
$\mathrm{~N}$ & $8.54_{-0.15}^{+0.11}$ & $7.79 \pm 0.04$ & 7.64 \\
$\mathrm{O}$ & $8.30_{-0.30}^{+0.18}$ & $8.76 \pm 0.05$ & 8.55 \\
\hline
\end{tabular}

can see that that the nitrogen abundance in $\mathrm{CPD}-64^{\circ} 2731$ is enhanced by a factor of 6-8 (see also Section 6 ).

\subsection{Reddening, distance and luminosity of CPD-64 2731}

To estimate the colour excess $E(B-V)$ towards CPD- $64^{\circ} 2731$, we compared the photometric measurements compiled in Table1 with the spectral energy distribution (SED) in the model spectrum. We found that the slopes of the observed and model spectra match each other if $E(B-V)=0.56 \mathrm{mag}$ (Fig. 4). Note that the derived value of $E(B-V)$ is equal to the full Galactic reddening in the direction to $\mathrm{CPD}-64^{\circ} 2731$ of $0.56 \mathrm{mag}$ (Schlafly \& Finkbeiner 2011), which is expectable in view of the high Galactic latitude location of the star. Note also that the same reddening could be derived by using the $B$ and $V$ photometry of $\mathrm{CPD}-64^{\circ} 2731$ and the intrinsic colour of the star of $(B-V)_{0}=-0.28 \mathrm{mag}$ (Martins \& Plez 2006), i.e. $E(B-V)=\left[(B-V)-(B-V)_{0}\right]=0.56 \mathrm{mag}$.

With the known reddening to $\mathrm{CPD}-64^{\circ} 2731$, one can estimate the distance to this star. Using the $V$-band absolute magnitude of an $05.5 \mathrm{~V}$ star of $M_{V}=-5.14$ mag (Martins $\&$ Plez 2006) and assuming the total-to-selective absorption ratio of $R=A_{V} / E(B-V)=3.1$, one finds the $V$-band extinction towards $\mathrm{CPD}-64^{\circ} 2731$ of $A_{V}=1.74 \mathrm{mag}$ and the distance modulus of this star of $\mathrm{DM}=V-M_{V}-A_{V}=14.00$ mag, implying a distance of $d \approx 6.3 \mathrm{kpc}$.

The obtained distance estimate should be compared with the parallactic distance based on the data from the Gaia second data release (DR2; Gaia Collaboration 2018). A simple inversion of the observed parallax of CPD-64 2731 of $0.091 \pm 0.026$ mas places this star at a distance of $d=10.95_{-2.42}^{+4.36} \mathrm{kpc}$. A shorter distance to CPD-64 2731 of $d=7.54_{-1.21}^{+1.67}$ was derived from the DR2 data by BailerJones et al. (2018) by using a Bayesian prior knowledge approach. This distance estimate agrees within the error margins with that based on the $M_{V}$-spectral type calibration.

To determine the luminosity, we recomputed the fluxes for three values of $d=6.3,7.5$ and $11 \mathrm{kpc}$. The resulting fluxes were corrected for the interstellar extinction, and then compared to the model spectra convolved with the transmission curves of the standard $B$ and $V$ filters. The obtained luminosity and other stellar parameters derived for three above values of $d$ are listed in Table 3 along with the surface abundances of main chemical elements (by mass). Note that at $d=6.3 \mathrm{kpc}(11 \mathrm{kpc})$ the inferred mass of 


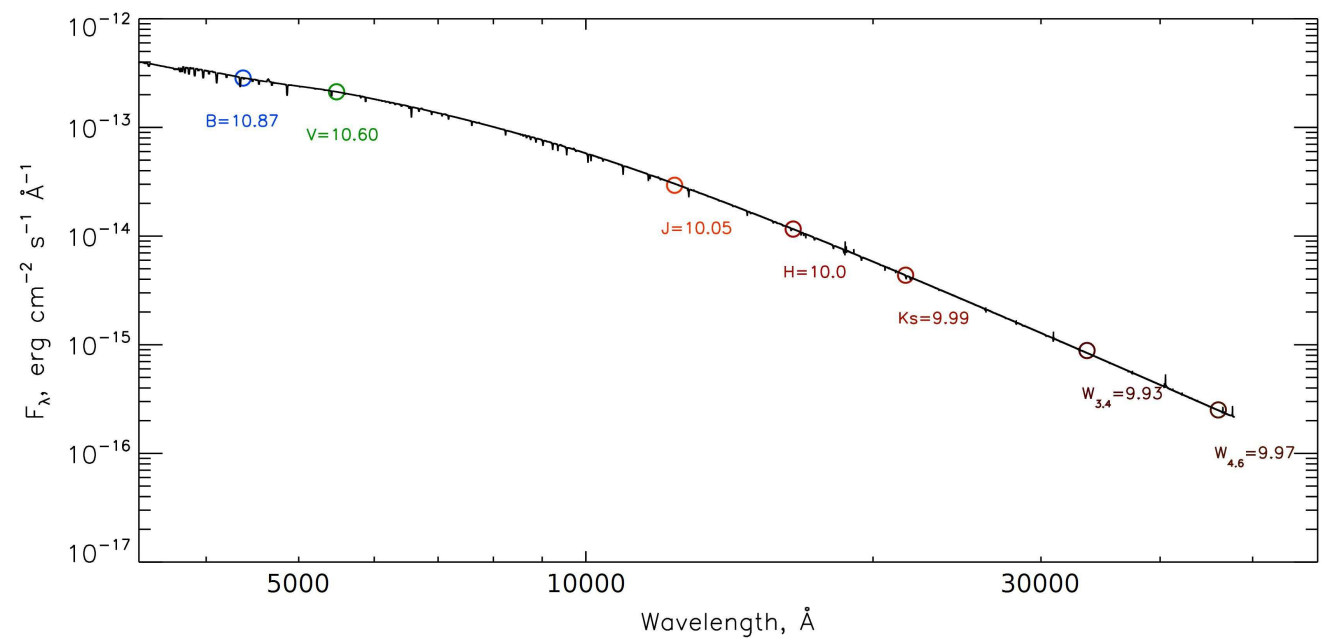

Figure 4. Observed flux distribution of CPD $-64^{\circ} 2731$ in absolute units, based on the photometric measurements (circles) compiled in Table1. compared to the emergent flux of the reddened model spectrum (black line) with the parameters as given in Table 3

Table 3. Stellar parameters of CPD $-64^{\circ} 2731$ for three distances (see text for details).

\begin{tabular}{lccc}
\hline$d(\mathrm{kpc})$ & 6.3 & 7.5 & 11 \\
$\log \left(L_{*} / \mathrm{L} \odot\right)$ & $5.40 \pm 0.03$ & $5.57 \pm 0.03$ & $5.93 \pm 0.01$ \\
$R_{*}(\mathrm{R} \odot)$ & 10 & 13 & 18 \\
$M_{*}(\mathrm{M} \odot)$ & 23 & 38 & 78 \\
$v_{\infty}\left(\mathrm{km} \mathrm{s}^{-1}\right)$ & 2500 & 2900 & 3400 \\
$\log \left(\dot{M} / \mathrm{M} \odot \mathrm{yr}^{-1}\right)$ & $-6.12 \pm 0.02$ & $-5.96 \pm 0.04$ & $-5.82 \pm 0.06$ \\
& & \\
$T_{\text {eff }}(\mathrm{kK})$ & $40.0 \pm 1.0$ & \\
$\log g$ & $3.8 \pm 0.1$ & \\
$\beta($ adopted $)$ & 0.8 & \\
$f$ (adopted) & 0.5 & \\
$v \sin i\left(\mathrm{~km} \mathrm{~s} \mathrm{~s}^{-1}\right)$ & $290_{-40}^{+20}$ & \\
$v_{\mathrm{h}, \text { hel }}\left(\mathrm{km} \mathrm{s} \mathrm{s}^{-1}\right)$ & $-175.7 \pm 0.3$ & \\
$\mathrm{H}($ mass fraction $)$ & $0.71 \pm 0.04$ & \\
$\mathrm{He}($ mass fraction $)$ & $0.28 \pm 0.04$ & \\
$\mathrm{C}$ (mass fraction) & $(1.7 \pm 0.9) \times 10^{-3}$ & \\
$\mathrm{~N}$ (mass fraction) & $(3.5 \pm 1) \times 10^{-3}$ & \\
$\mathrm{O}($ mass fraction) & $(2.3 \pm 1.1) \times 10^{-3}$ & \\
$E(B-V)$ (mag) & 0.56 & \\
\hline
\end{tabular}

$\mathrm{CPD}-64^{\circ} 2731$ of $23 \mathrm{M} \odot(78 \mathrm{M} \odot)$ is much smaller (larger) than what one might expect for an unevolved O5.5 star (cf. Martins, Schaerer \& Hillier 2005; Weidner \& Vink 2010). Proceeding from this, in what follows we adopt the distance to $\mathrm{CPD}-64^{\circ} 2731$ of $7.5 \mathrm{kpc}$, and note that the main conclusions of the paper do not change if the actual distance to the star is within reasonable limits around this value.

\subsection{Rotational and radial velocities}

The high resolution of the HRS spectra allowed us to determine the projected rotational velocity of CPD-64 2731 and to search for possible radial velocity variability of this star.

The projected rotational velocity was derived through the Fourier transform of the He I $\lambda 4471$ line profile using the IACOB-BROAD code (Simón-Díaz \& Herrero 2007, 2014). The obtained value of $v \sin i \approx 290_{-40}^{+20}$, where $i$ is the angle between the stellar rotation axis and our line-of-sight, turns out to be extremely high for a O5.5 star (we further discuss this point in Section 6).

The heliocentric radial velocity, $v_{\mathrm{r}, \text { hel }}$, of CPD-6 $4^{\circ} 2731$ given in Table 3 is the mean value based on three HRS spectra. The measurements were done with a dedicated software package developed by our team (Katkov et al., in preparation). This software is based on the library of high-resolution theoretical stellar spectra (Coelho 2014) and is designed to derive radial velocities for components of binary systems. We simultaneously fitted the available observed spectra using the same model spectrum interpolated from the grid of stellar models and convolved with the instrumental resolution and $v \sin i$, and shifted with individual line-of-sight velocity for a given epoch. In clear cases of binary stars the package uses two model spectra with individual velocities. We did not reveal any signature of a second star in the spectra of $\mathrm{CPD}-64^{\circ} 2731$, and therefore measured only one radial ve- 


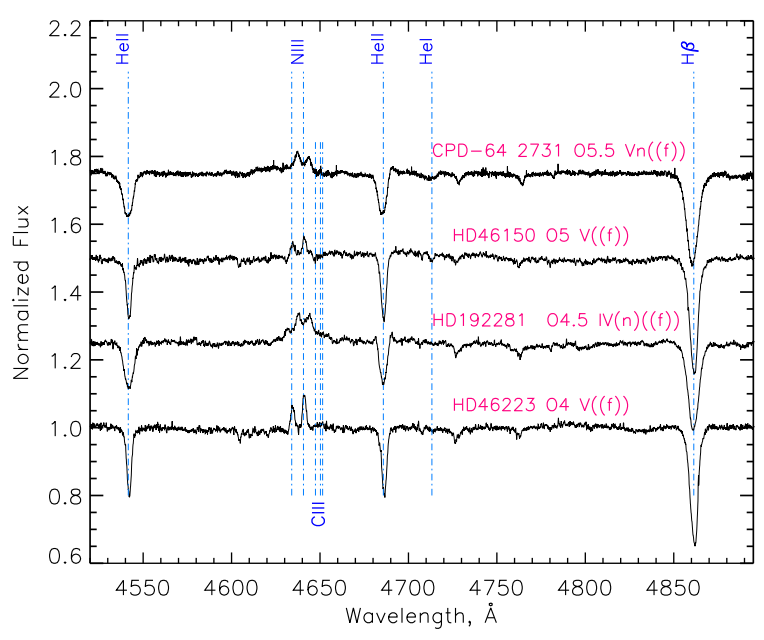

Figure 5. Comparison of a portion of the HRS spectrum of CPD $-64^{\circ} 2731$ with the corresponding parts of spectra of three other $\mathrm{O}((\mathrm{f}))$ stars. Note that the N III lines in the spectra of CPD $-64^{\circ} 2731$ and HD 192281 are shifted redwards.

locity for each analysed spectrum. The obtained values of $v_{\mathrm{r}, \text { hel }}$ of $-178.6 \pm 0.4 \mathrm{~km} \mathrm{~s}^{-1}$ (2018 February 1), $-174.9 \pm$ $0.6 \mathrm{~km} \mathrm{~s}^{-1}$ (2018 February 25) and $-173.7 \pm 0.6 \mathrm{~km} \mathrm{~s}^{-1}$ (2018 July 18) indicate that CPD $-64^{\circ} 2731$ might be a wide binary system (cf. Section 6), unless the uncertainties in the radial velocity measurements are underestimated due to possible unaccounted systematic errors.

Finally, we note that the only emission lines detected in the spectrum of CPD-64 2731 , namely the $\mathrm{N}$ III $\lambda \lambda 4634$, 41-42 ones, are shifted redwards with respect to the absorption lines by $\approx 4 \AA$ or $\approx 260 \mathrm{~km} \mathrm{~s}^{-1}$. Similar shift of the same lines was also found in the spectrum of the O4.5 IV(n)((f)) (Maíz Apellániz et al. 2016) star HD 192281 (Sota et al. 2014), as illustrated in Fig.5, where we compare a portion of the spectrum of CPD-64 2731 with the corresponding parts of spectra of HD 192281 and two other $\mathrm{O}((\mathrm{f}))$ stars (retrieved from the ELODIE archive at Observatoire de Haute-Provence; Moultaka et al. 2004). Sota et al. (2014) suggested that the shift of the N III lines in the spectrum of HD 192281 is caused by the binarity of this star, which is also responsible for the line broadening in the spectrum. The possible binary status of HD 192281 was first suggested by Barannikov (1993) who found small-amplitude radial velocity variability with a period of 5.48 day. More recent measurements by Cazorla et al. (2017) also found evidence for variability, but did not confirm its periodicity, meaning that HD 192281 might not necessarily be a binary system. Since the N III lines are produced in the stellar photosphere (Brucato \& Mihalas 1971), we speculate that their shift is somehow related to asymmetry of the photosphere, probably caused by the fast rotation and/or strong magnetic field (expected in the star if it is a binary merger product; cf. Section 6). Spectropolarimetric observations of CPD $-64^{\circ} 2731$ could be of high importance to clarify this issue.

\section{CPD-64 2731 AS A RUNAWAY STAR}

The Gaia DR2 provides high-precise proper motion measurements for $\mathrm{CPD}-64^{\circ} 2731$ (see Table 4): $\mu_{\alpha} \cos \delta=$ $-6.69 \pm 0.03$ mas yr$^{-1}$ and $\mu_{\delta}=-4.83 \pm 0.04$ mas yr$^{-1}$. Using these data and $v_{\mathrm{r}, \text { hel }}$ from Table 3 we calculated the peculiar transverse velocity $v_{\mathrm{tr}}=\left(v_{1}^{2}+v_{\mathrm{b}}^{2}\right)^{1 / 2}$, where $v_{1}$ and $v_{\mathrm{b}}$ are, respectively, the peculiar velocity components along the Galactic longitude and latitude, the peculiar radial velocity $v_{\mathrm{r}}$, and the total space velocity $v_{*}$ of the star. For this, we adopted the solar Galactocentric distance of $R_{0}=8.0 \mathrm{kpc}$ and the circular Galactic rotation velocity of $\Theta_{0}=240 \mathrm{kms}^{-1}$ (Reid et al. 2009), and the solar peculiar motion of $\left(U_{\odot}, V_{\odot}, W_{\odot}\right)=(11.1,12.2,7.3) \mathrm{kms}^{-1}$ (Schönrich, Binney \& Dehnen 2010). For the error calculation, only the errors of the proper motion and the radial velocity measurements were considered. For the sake of completeness, we calculated velocities for three value of distance: $d=6.3,7.5$ and $11 \mathrm{kpc}$. The results are given in Table 4 along with the separation of CPD- $64^{\circ} 2731$ from the Galactic plane, $z=d \sin b$, and the kinematic age of this star, $t_{\mathrm{kin}}=z / v_{\mathrm{b}}$.

From Table 4 it follows that $\mathrm{CPD}-64^{\circ} 2731$ is a highvelocity runaway star. The runaway status of $\mathrm{CPD}-64^{\circ} 2731$ is also supported by the large separation of this star from the Galactic plane (cf. Blaauw 1961; van Oijen 1989), which is much larger than the scale heights of runaway $\mathrm{O}$ and 'normal' OB stars of, respectively, $\approx 100$ pc (Stone 1979) and $\approx 50 \mathrm{pc}$ (Stone 1979; Reed 2000).

At the adopted distance of $d=7.5 \mathrm{kpc}$, the space velocity of $\mathrm{CPD}-64^{\circ} 2731$ is $\approx 160 \mathrm{~km} \mathrm{~s}^{-1}$, the star is approaching us with a velocity of $\approx 140 \mathrm{~km} \mathrm{~s}^{-1}$, and the vector of its total space velocity is inclined to our line of sight by $\approx 33^{\circ}$. Although a $40 \mathrm{M} \odot$ star can, in principle, attain a velocity of $160 \mathrm{~km} \mathrm{~s}^{-1}$ in the course of dissolution of a close binary system following asymmetric supernova explosion (e.g. Portegies Zwart 2000; Eldridge, Langer \& Tout 2011), the young age of $\mathrm{CPD}-64^{\circ} 2731$ ( $\lesssim 3 \mathrm{Myr}$; see next section) and the high rotational velocity of this star make this possibility unlikely (see below). This, along with observations that massive stars form in a clustered way (Lada \& Lada 2003; Gvaramadze et al. 2012b), indicates that CPD-64 2731 attained its high space velocity because of a three- or fourbody dynamical encounter with other massive stars in the parent star cluster (Poveda, Ruiz \& Allen 1967; Leonard \& Duncan 1990). Furthermore, the high mass and velocity of $\mathrm{CPD}-64^{\circ} 2731$ imply that the kinetic energy of this star is one of the largest among known runaway star\$2, and that the stars involved in the dynamical interaction were much more massive than this star.

For example, numerical experiments show (see figs 7 and 11 in Gvaramadze \& Gualandris 2011) that a $40 \mathrm{M} \odot$ star could be accelerated to a velocity of $\geqslant 160 \mathrm{~km} \mathrm{~s}^{-1}$ in the course of encounter with a massive binary of total mass similar to that of the most massive known binaries in the Milky Way, WR 20a and NGC 3603-A1, whose masses are, respectively, $\approx 160$ and $200 \mathrm{M} \odot$. Both WR 20a and NGC 3603A1 are members of young, very massive $\left(\sim 10^{4} \mathrm{M} \odot\right)$ star

2 Two other massive runaway stars with record kinetic energy are the $\mathrm{O} 2$ stars $\mathrm{Sk}-67^{\circ} 22$ and BI 237 in the Large Magellanic Cloud (Massey et al. 2005). 
Table 4. Astrometric and kinematic data on $\mathrm{CPD}-64^{\circ} 2731$ (see text for details).

\begin{tabular}{cccccccc}
\hline $\begin{array}{c}d \\
(\mathrm{kpc})\end{array}$ & $\begin{array}{c}v_{\mathrm{l}} \\
\left(\mathrm{km} \mathrm{s}^{-1}\right)\end{array}$ & $\begin{array}{c}v_{\mathrm{b}} \\
\left(\mathrm{km} \mathrm{s}^{-1}\right)\end{array}$ & $\begin{array}{c}v_{\mathrm{r}} \\
\left(\mathrm{km} \mathrm{s}^{-1}\right)\end{array}$ & $\begin{array}{c}v_{\mathrm{tr}} \\
\left(\mathrm{km} \mathrm{s}^{-1}\right)\end{array}$ & $\begin{array}{c}v_{*} \\
\left(\mathrm{~km} \mathrm{~s}^{-1}\right)\end{array}$ & $\begin{array}{c}z \\
(\mathrm{pc})\end{array}$ & $\begin{array}{c}t_{\mathrm{kin}} \\
(\mathrm{Myr})\end{array}$ \\
\hline 6.3 & $-18.2 \pm 1.0$ & $-70.4 \pm 1.1$ & $-125.2 \pm 0.3$ & $72.7 \pm 1.1$ & $144.8 \pm 0.6$ & 410 & 5.9 \\
7.5 & $-20.4 \pm 1.2$ & $-86.6 \pm 1.3$ & $-136.0 \pm 0.3$ & $89.0 \pm 1.3$ & $162.5 \pm 0.8$ & 490 & 5.7 \\
11 & $-68.3 \pm 1.8$ & $-134.2 \pm 1.9$ & $-186.2 \pm 0.3$ & $150.6 \pm 1.5$ & $239.5 \pm 1.0$ & 720 & 5.4 \\
\hline
\end{tabular}

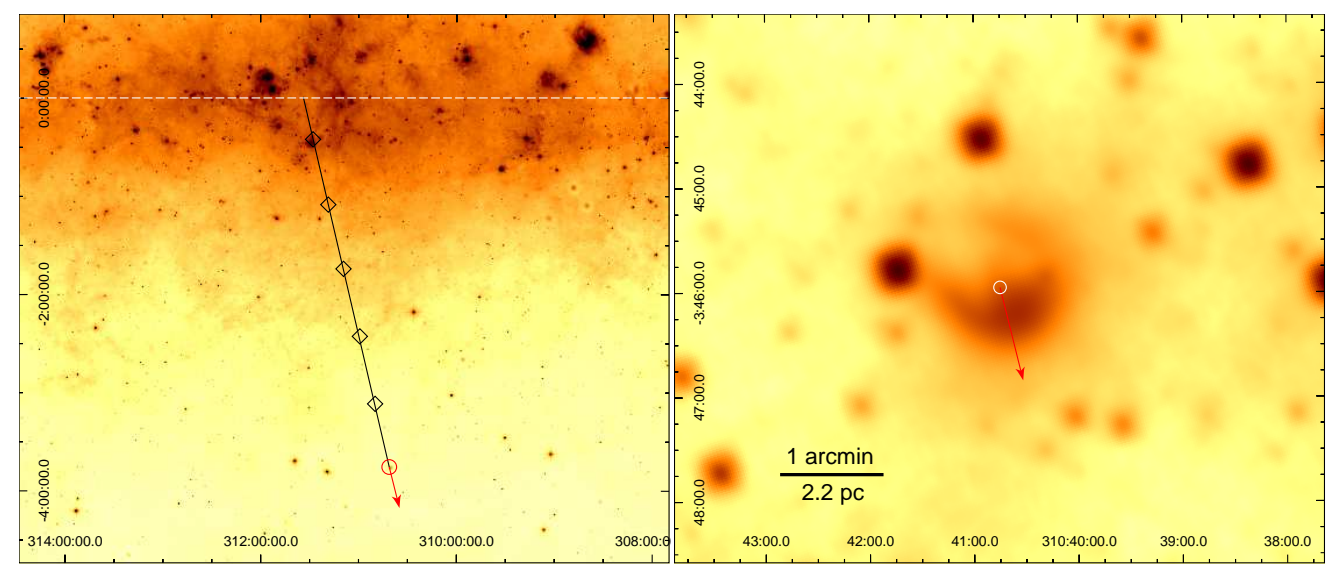

Figure 6. Left-hand panel: WISE $22 \mu \mathrm{m}$ image of the field containing CPD- $64^{\circ} 2731$ and its surrounding nebula (marked by a circle). The arrow shows the direction of peculiar transverse velocity of CPD-64을 2731 , derived using the Gaia DR2 proper motion measurement and adopting a distance to this star of $7.5 \mathrm{kpc}$. The solid (black) line shows the trajectory of CPD $-64^{\circ} 2731$, and diamonds mark the positions of the star 1, 2, 3, 4 and $5 \mathrm{Myr}$ ago. The Galactic plane is indicated by a white dashed line. Right-hand panel: WISE $12 \mu \mathrm{m}$ image of the nebula with position of CPD $-64^{\circ} 2731$ marked by a circle. The arrow shows the direction of peculiar transverse velocity of the star. In both panels, the coordinates (in units of degrees) are the Galactic longitude and latitude on the horizontal and vertical scales, respectively. At a distance of $7.5 \mathrm{kpc}, 1^{\circ}$ and 1 arcmin correspond, respectively, to $\approx 130$ and 2.2 pc. See the text for details.

clusters, which, like other star clusters of this mass, are located in the Galactic plane. It is likely, therefore, that CPD $-64^{\circ} 2731$ was ejected from a similar very massive cluster in the Galactic plane.

Figure 6 shows that $\mathrm{CPD}-64^{\circ} 2731$ is moving almost perpendicular to the Galactic plane and that its past trajectory intersects the Galactic plane at $l \approx 311^{\circ} .5$. Note that the orientation of the transverse peculiar velocity of the star only slightly depends on the distance; by varying the distance between 6 and $9 \mathrm{kpc}$, we found that the corresponding past trajectories of $\mathrm{CPD}-64^{\circ} 2731$ intersect the Galactic plane within a half degree from each other. In this direction our line of sight first crosses the Carina-Sagittarius arm at $\approx 1.5 \mathrm{kpc}$ and then tangentially enters into the Crux-Scutum spiral arm at $\approx 5 \mathrm{kpc}$ (Cordes \& Lazio 2002). All distance estimates for CPD-64 2731 imply that this star was formed in the Crux-Scutum arm.

We searched for known young ( $\leqslant 10 \mathrm{Myr})$ star clusters in the region between Galactic longitudes $310^{\circ}$ and $313^{\circ}$ using the WEBDA data bas屯 3 (Mermilliod 1995), but did not find any. This non-detection may indicate that the parent cluster to $\mathrm{CPD}-64^{\circ} 2731$ is already dissolved following residual-gas expulsion at the very beginning of cluster dynamical evolution (Tutukov 1978) or is heavily obscured by the foreground dust in the Galactic plane. Note that the very massive binary involved in the dynamical interaction with $\mathrm{CPD}-64^{\circ} 2731$

\footnotetext{
3 http://webda.physics.muni.cz/
}

and recoiled in the opposite direction to this star should have ended its life in supernova explosions several Myr ago.

\section{CPD- $64^{\circ} 2731$ AS A SPUN-UP AND REJUVENATED STAR}

Figure 7 shows the position of $\mathrm{CPD}-64^{\circ} 2731$ in the Hertzsprung-Russell diagram along with evolutionary tracks for Galactic non-rotating and rotating $\mathrm{O}$ stars by Brott et al. (2011). From the figure it follows that if CPD-64 2731 was born as a single star, then its initial mass, $M_{\text {init }}$, was between 40 and $50 \mathrm{M} \odot$.

In Table 5 we compile the main parameters of CPD $-64^{\circ} 2731$ and the corresponding parameters of several 40 and $50 \mathrm{M} \odot$ model stars from Brott et al. (2011) for the times when their effective temperatures decrease to $40 \mathrm{kK}$. One can see that in the non-rotating model star (model1) the CNO abundances remain almost equal to the initial abundances adopted in Brott et al. (2011). With the increase of the initial rotational velocity, $v_{\text {init }}$, the nitrogen abundance in the model stars also grows and becomes comparable to that in $\mathrm{CPD}-64^{\circ} 2731$ for $v_{\text {init }} \approx 400 \mathrm{~km} \mathrm{~s}^{-1}$ (models 3 and 5). For this $v_{\text {init }}$ the oxygen abundance in the model stars also agrees with that derived for $\mathrm{CPD}-64^{\circ} 2731$, while the carbon abundance is much lower, which in part could be due to low initial abundance of this element adopted in the models (see Table2), and in part due to inaccuracy of our spectral modelling. For higher values of $v_{\text {init }}$ (model 4 ), 
Table 5. Comparison of CPD-64 2731 with model stars from Brott et al. (2011).

\begin{tabular}{lcccccc}
\hline & CPD -64 2731 & Model 1 & Model 2 & Model 3 & Model 4 & Model 5 \\
\hline$M_{\text {init }}(\mathrm{M} \odot)$ & - & 40 & 40 & 40 & 40 & 50 \\
$v_{\text {init }}\left(\mathrm{km} \mathrm{s}^{-1}\right)$ & - & 0 & 317 & 367 & 464 & 364 \\
age $(\mathrm{Myr})$ & - & 2.1 & 2.15 & 2.8 & 4.0 & 2.4 \\
$M_{*}(\mathrm{M} \odot)$ & 38 & 38.4 & 38.2 & 37.4 & 34.9 & 45.4 \\
$T_{\text {eff }}(\mathrm{kK})$ & $39.5-40.5$ & 40 & 40 & 40 & 40 & 40 \\
$\log \left(L_{*} / \mathrm{L} \odot\right)$ & $5.54-5.60$ & 5.47 & 5.45 & 5.49 & 5.58 & 5.68 \\
$R_{*}(\mathrm{R} \odot)$ & 13 & 11 & 11 & 12 & 13 & 14 \\
$\log g$ & $3.7-3.9$ & 3.9 & 3.9 & 3.9 & 3.8 & 3.8 \\
$v_{\text {surf }}\left(\mathrm{km} \mathrm{s}^{-1}\right)$ & $250-310^{a}$ & 0 & 283 & 300 & 282 & 275 \\
$\mathrm{He}($ mass fraction $)$ & $0.24-0.32$ & 0.26 & 0.31 & 0.30 & 0.27 & 0.28 \\
$\mathrm{C}$ (mass fraction) $\left(10^{-3}\right)$ & $0.80-2.60$ & 1.18 & 0.63 & 0.23 & 0.08 & 0.32 \\
$\mathrm{~N}$ (mass fraction) $\left(10^{-3}\right)$ & $2.50-4.50$ & 0.44 & 1.84 & 3.68 & 5.02 & 3.14 \\
$\mathrm{O}$ (mass fraction) $\left(10^{-3}\right)$ & $1.20-3.40$ & 4.13 & 3.29 & 1.71 & 0.37 & 2.20 \\
\hline
\end{tabular}

Note. ${ }^{a} v \sin i$.

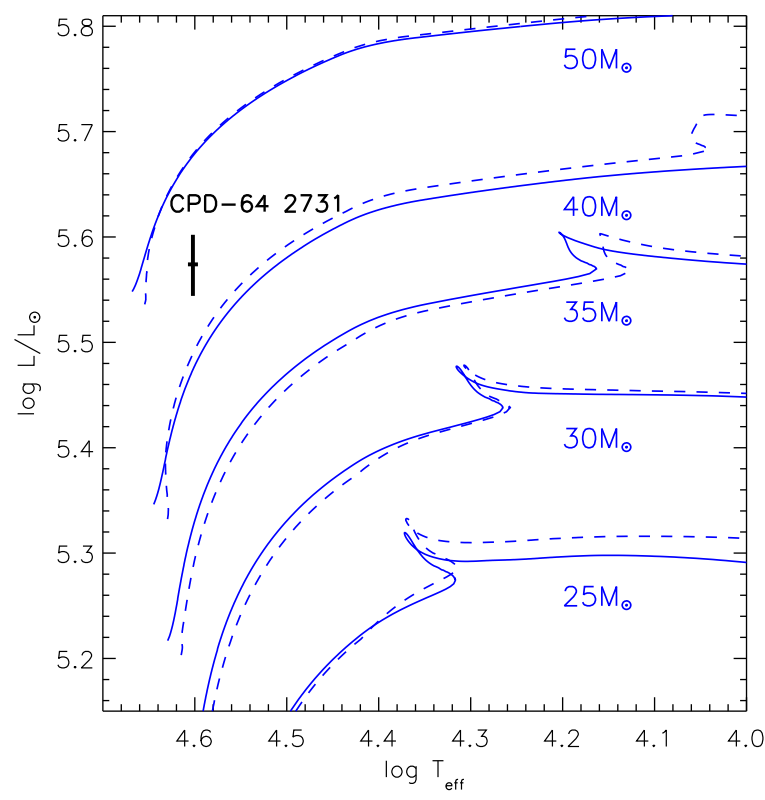

Figure 7. Position of CPD-64을 2731 in the Hertzsprung-Russell diagram (cross with error bars). Also shown are the evolutionary tracks of Brott et al. (2011) for Galactic O stars. The dashed and solid lines correspond, respectively, to the tracks with initial rotational velocities of 0 and $\approx 400 \mathrm{~km} \mathrm{~s}^{-1}$.

the model star remains hotter than $40 \mathrm{kK}$ for a longer time, and, correspondingly, its $\mathrm{N}$ abundance increases even more, while the $\mathrm{C}$ and $\mathrm{O}$ abundances become much more depleted as compared to those in $\mathrm{CPD}-64^{\circ} 2731$.

More importantly, Table 5 shows that if CPD-64 2731 underwent effectively single-star evolution then its age should be $\lesssim 3 \mathrm{Myr}$, i.e. a factor of two younger than the kinematic age (see Table 4). This implies that CPD-64 2731 could be a rejuvenated and spun-up product of binary mass transfer or the result of a binary merger, i.e., a massive blue straggler. This in turn implies that $\mathrm{CPD}-64^{\circ} 2731$ was initially a compact binary system, which was ejected via strong few-body dynamical interaction (e.g. Oh \& Kroupa 2012). Numerical scattering experiments by Kroupa (1998) showed that a binary system can survive the ejection process only if its space velocity is less than the orbital velocity. These experiments also showed that the higher ejection velocity of runaway binaries the closer their mass ratio to unity.

Let us assume that the progenitor binary of $\mathrm{CPD}-64^{\circ} 2731$ was composed of two $\approx 20 \mathrm{M} \odot$ stars. Such a binary would undergo stable mass transfer if its initial orbital period was $\gtrsim 3 \mathrm{~d}$ (Wellstein, Langer \& Braun 2001) or merger if the orbital period was shorter and the masses of the binary components were equal to each other (cf. Schneider et al. 2015). The enhanced nitrogen abundance in $\mathrm{CPD}-64^{\circ} 2731$ could be the direct result of binary mass transfer (Langer 2012) or of merger process of two stars (Glebbeek et al. 2013). The merger ends with a single star, while in the case of mass transfer one would expect a helium star companion in a wide $(\approx 30 \mathrm{~d})$ orbit around $\mathrm{CPD}-64^{\circ} 2731$, which can be quite faint at optical wavelengths to be detected (e.g. Götberg, de Mink \& Groh 2017). In the latter case, $\mathrm{CPD}-64^{\circ} 2731$ should show a radial velocity variation of $\approx 20 \mathrm{~km} \mathrm{~s}^{-1}$ (Wellstein et al. 2001). Since the existing data do not allow us to firmly assert that $\mathrm{CPD}-64^{\circ} 2731$ is a binary system, we reserve the possibility that this star could be a binary merger product.

The high projected rotational velocity of $\mathrm{CPD}-64^{\circ} 2731$ supports the possibility that this star is a product of binary evolution. Indeed, observations of $\approx 200$ Galactic OB stars show that the distribution of their projected rotational velocities peaks at $\approx 50 \mathrm{~km} \mathrm{~s}^{-1}$ and that only few of them have velocities of $\gtrsim 300 \mathrm{~km} \mathrm{~s}^{-1}$ (Simón-Díaz \& Herrero 2014). Furthermore, only two of these fast-rotating stars are of spectral type earlier than O6. Similarly, the study of projected rotational velocities of $\approx 200$ apparently single $O$ stars in the 30 Doradus star forming region of the Large Magellanic Cloud by Ramírez-Agudelo et al. (2013) shows that none of the early O-type $(\mathrm{O} 2-5)$ stars rotate faster than $300 \mathrm{~km} \mathrm{~s}^{-1}$. The lack of fast rotators among the early O-type stars could be explained by removal of angular momentum of their surface layers through wind mass loss, which is more efficient for massive stars because of their strong winds (e.g. Langer 1998). On the other hand, the existence of the highvelocity tail in the distribution of rotational velocities could be understood if it is populated by products of binary interaction, e.g. by spun-up mass gainers or merged binaries (Sana et al. 2012; de Mink et al. 2013). 
Interestingly, observations also show that the fraction of fast-rotating O stars is larger outside star clusters (RamírezAgudelo et al. 2013; see their fig. 12). Ramírez-Agudelo et al. (2013) suggested that the fast rotators in the field may be spun-up post-binary interaction products that were ejected from parent clusters when their primary stars exploded in supernovae. Alternatively, the rapid rotation of at least some of the field $\mathrm{O}$ stars could be explained by mass transfer in or merger of runaway binaries (recall that most runaways are binary systems, meaning that they were ejected in the field because of dynamical few-body interactions; Chini et al. 2012). This possibility is supported by the results of numerical modelling of young massive star clusters, which show that the orbital period distribution of runaway binaries is biased towards shorter periods as compared to binaries retained in the clusters (Oh \& Kroupa 2016), and that the runaway binaries tend to have eccentric orbits (Leonard \& Duncan 1990), implying that they are more prone for binary interaction processes.

Finally, we point out that within the merger scenario, the high rotational velocity of $\mathrm{CPD}-64^{\circ} 2731$ serves as an indication that the merger having occurred very recently. Indeed, if the origin of magnetic field in massive stars is due to binary merger (e.g. Ferrario et al. 2009; Langer 2012; see also Section 9) then the spin-down of the merger product is extremely fast, such that most magnetic stars rotate very slowly (Fossati et al. 2016).

\section{THE ORIGIN OF THE NEBULA}

The high space velocity of $\mathrm{CPD}-64^{\circ} 2731$ implies that the ram pressure of the ISM should play important role in shaping the nebula. This inference is supported by the orientation of the peculiar transverse velocity vector, which is pointed towards the brightest side of the nebula, and by the offset of the star from the centre of the nebula in the same direction. Below we propose a possible scenario for the origin of the nebula.

First, we note that the nebula hardly can be explained in the framework of single star evolution. Indeed, the unevolved status of $\mathrm{CPD}-64^{\circ} 2731$ excludes the possibility that the nebula was formed because of the wind-wind interaction at the advanced stages of stellar evolution, as it is the case for circumstellar nebulae around evolved massive stars (e.g. García-Segura et al. 1996a,b; Brighenti \& D'Ercole 1997). Also, the high temperature of CPD-6 $4^{\circ} 2731$ implies that the nebula cannot be formed during an episode of enhanced mass loss caused by the bi-stability jump - a factor of 10 increase of the mass-loss rate when the stellar temperature drops below a critical value of $\approx 21 \mathrm{kK}$ (Pauldrach \& Puls 1990; Vink 2018). Furthermore, the general appearance of the nebula and the nearly central location of CPD-64 2731 within it allow us to argue that the nebula is not a genuine bow shock (we validate this assertion in Section 8 by means of numerical modelling).

We concluded above that $\mathrm{CPD}-64^{\circ} 2731$ is either a mass gainer from a binary mass transfer or a merger star. In both cases, a few solar masses of material are likely spilled and blown into the circumstellar environment with rather small velocity (e.g., Petrovic et al. 2005; Glebbeek et al. 2013). Therefore, the nebula could be caused by changes in the mass-loss rate and wind velocity following the binary interaction. We suggest the following scenario.

Originally, $\mathrm{CPD}-64^{\circ} 2731$ was a (runaway) binary system composed of two almost equal-mass stars. The combined winds from these stars created a bow shock ahead of the system. The stand-off distance of the bow shock, i.e. the distance between the stars and the nearest side of the bow shock (apex), is given by (Baranov, Krasnobaev \& Kulikovskii 1970)

$R_{0}=\left(\frac{\dot{M} v_{\infty}}{4 \pi \rho_{\mathrm{ISM}} v_{*}^{2}}\right)^{1 / 2}$,

where $\rho_{\mathrm{ISM}}=1.4 m_{\mathrm{H}} n_{\mathrm{ISM}}, m_{\mathrm{H}}$ is the mass of a hydrogen atom, and $n_{\text {ISM }}$ is the number density of the local ISM. At some point, the binary interaction starts. This leads to a strong and slow wind, either from the fast rotating accretion star or from the bloated merger product. In both cases, this wind is expected to last for a Kelvin-Helmholtz timescale (about several thousand years), after which the binary product will establish a fast wind started to blow a bubble in the ambient medium. The geometry of the bubble is affected by the presence of shocked ISM ahead of the star and the bubble initially looks like a horseshoe-shaped nebula with its open ends pointing downstream and the star located close to the geometric centre of the nebula. We suggest that currently we are witnessing just this situation and that later on, after the star will completely overrun the preprocessed ISM, the bubble will transform into a classical bow shock. To validate this scenario, we performed numerical simulations, as described and discussed in the next section.

Our scenario for the formation of the observed circumstellar nebula around $\mathrm{CPD}-64^{\circ} 2731$ has the implication that the strong binary interaction happened only recently. This in turn strongly supports the idea that CPD- $64^{\circ} 2731$ was dynamically ejected from a dense star cluster as a binary system, and argues against the possibility that it was ejected from a broken-up binary as a consequence of the explosion of its companion.

\section{NUMERICAL SIMULATIONS}

To model the interaction between the stellar wind and the ISM, we used the time-dependent Godunov scheme (Godunov et al. 1979), which was applied to a curvilinear moving grid. The discontinuities in the flows were allocated by using the fitting-capturing technique (Myasnikov 1997; Izmodenov \& Alexashov 2015). For simplification of the numerical simulations, we used the shock capturing method for the inner (stellar wind) shock, while for the outer (bow) shock and the contact/tangential discontinuities the exact fitting was applied. When necessary, the outer boundary of the grid was also moving.

To increase the resolution properties of the first order Godunov scheme a piecewise-linear distribution of the parameters inside the grid cells were constructed. To achieve the TVD (Total Variation Diminishing) property of the scheme the slope limiters with compression parameters were used (Chakravarthy \& Osher 1985). The resolution in time remained first order which is not critical because of the small time step. The applied numerical method conserves the number of cells between discontinuities (in shock layers) 


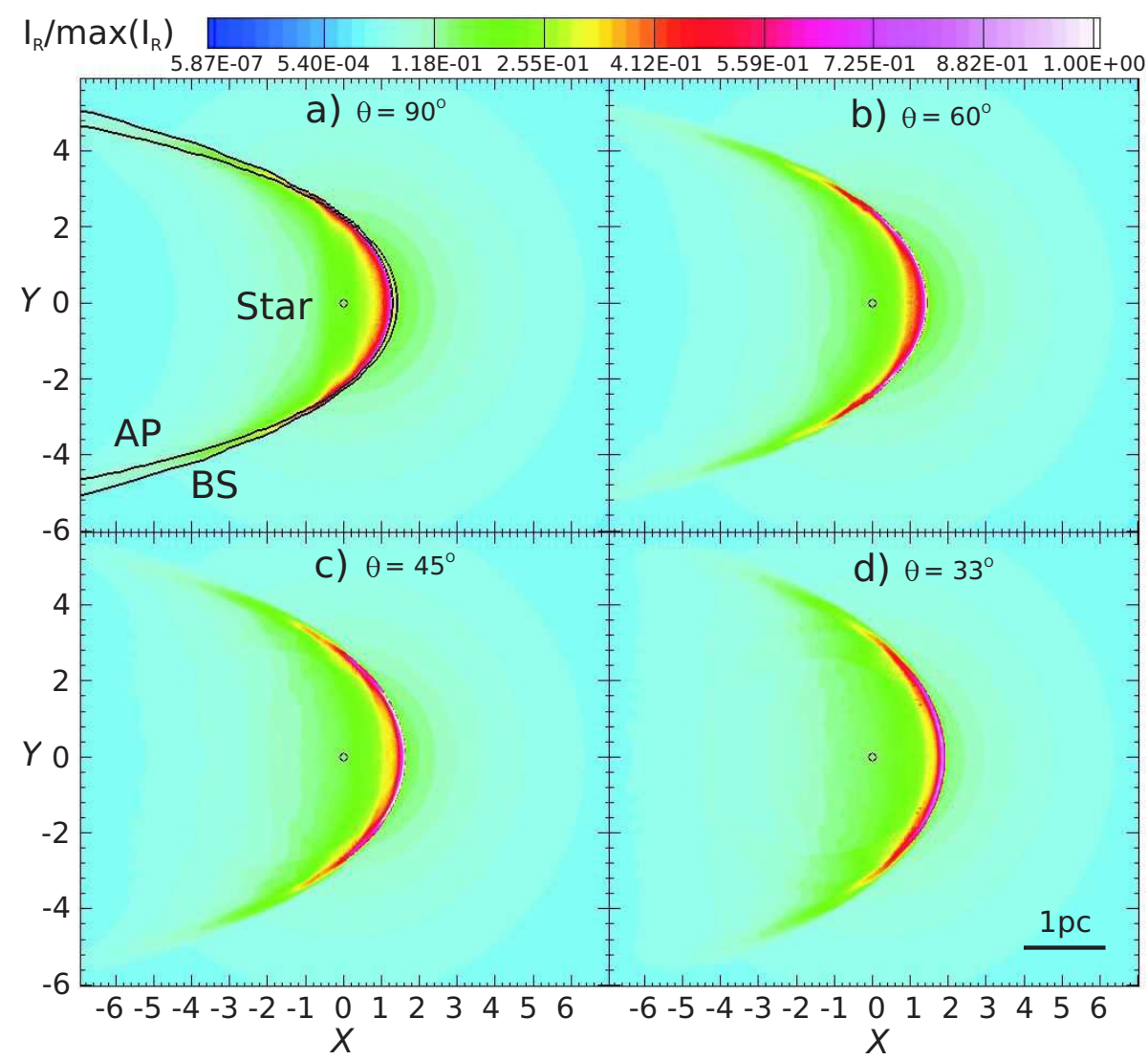

Figure 8. Synthetic maps of $22 \mu \mathrm{m}$ dust emission (normalized to the maximum surface brightness of panel d) from the model bow shock at four inclination angles of the line of sight to the symmetry axis of the simulation $(Y=0)$ : a) $90^{\circ}$, b) $60^{\circ}$, c) $45^{\circ}$, and d) $33^{\circ}$. The wind parameters of the wind-blowing star are equal to those of $\mathrm{CPD}-64^{\circ} 2731$. Solid lines in panel a) show the positions of astropause (AP) and bow shock (BS). The distance units on the $X$ and $Y$ axes are in 0.45 pc.

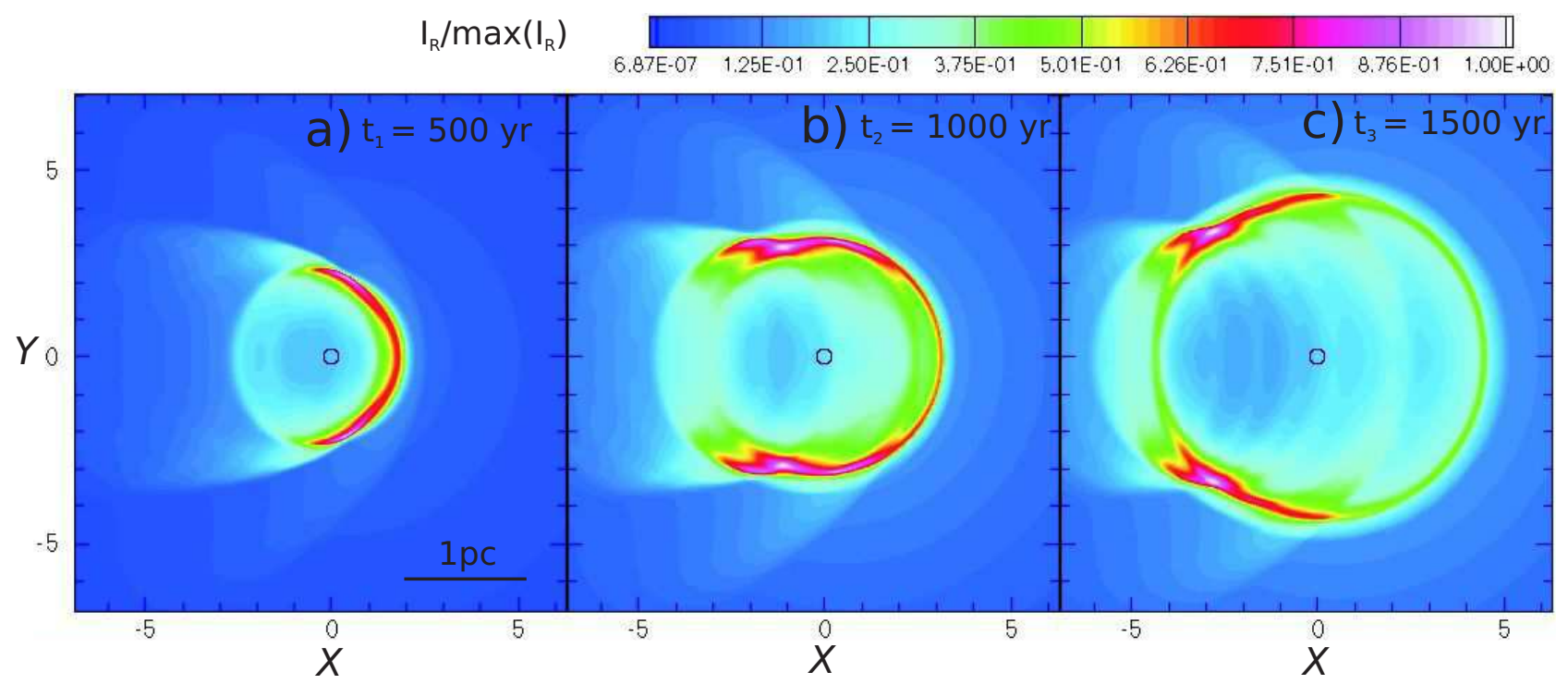

Figure 9. Synthetic maps of $22 \mu \mathrm{m}$ dust emission (normalized to the maximum surface brightness of each panel) from the shell driven by the variable wind from the binary product at three times and for the inclination angle of $33^{\circ}$. The distance units on the $X$ and $Y$ axes are in $0.3 \mathrm{pc}$. 
and, consequently, conserves the necessary resolution in thin shock layers. This is important for the numerical accuracy, e.g., when the radiative losses are taken into account (in our simulations we used the cooling function from Cowie, McKee \& Ostriker 1981). The simulations were performed in the reference frame of the star on a $2 \mathrm{D}$ grid with $200 \times 200$ cells. For verification of numerical solutions the resolution was increased up to two times.

To produce synthetic maps of dust emission, we assumed that the dust grains are heated only due to absorption of the stellar radiation, which is re-emitted as a black body radiation. In this case, the temperature of the dust is related to the temperature and radius of the star as follows: $T_{\mathrm{d}}(r)=T_{*}\left(R_{*} / 2 r\right)^{(2 / 4+\beta)}$, where $r$ is the distance from the star, and the parameter $\beta$ ranges between 1 and 2 , depending on the properties of the dust grains (see, e.g., Decin et al. 2012 and references therein). We also assumed that dust exist only in the ISM and the material of the slow wind. We run simulations for two limiting values $\beta$ and found that the choice of a particular value affects mainly the magnitude of the surface brightness. For the sake of definiteness, in what follows we present the results obtained for $\beta=2$. Since the main goal of our simulations is to show that the general shape of the nebula cannot be explained in the framework of the bow shock scenario and because most of the input parameters (such as $n_{\mathrm{ISM}}$ and the wind parameters before and after the binary interaction) are unknown, we did not try to reproduce the absolute surface brightness of the nebula. Instead, in the synthetic dust emission maps we show the projected surface brightness normalized to the maximum value.

In our modelling we assumed that the wind-blowing star is moving with a velocity of $160 \mathrm{~km} \mathrm{~s}^{-1}$ through the homogeneous ISM of temperature $8000 \mathrm{~K}$, and that there is no large-scale motions in the ISM. First, we modelled a bow shock produced by a star whose wind parameters $(\dot{M}$ and $\left.v_{\infty}\right)$ are identical to those of $\mathrm{CPD}-64^{\circ} 2731$. We run this simulation to show that the nebula around $\mathrm{CPD}-64^{\circ} 2731$ cannot be interpreted as a bow shock. For this modelling we used the number density of the ISM of $\approx 0.4 \mathrm{~cm}^{-3}$, which follows from equation (1) and the observed value of $R_{0}$ of $\approx 0.9 \mathrm{pc}$.

To verify our scenario for the origin of the nebula, we also run a second simulation in which the runaway star was assumed to be initially composed of two $\approx 20 \mathrm{M} \odot$ components with identical $\dot{M}$ and $v_{\infty}$ of, respectively, $10^{-8} \mathrm{M} \odot \mathrm{yr}^{-1}$ and $1200 \mathrm{~km} \mathrm{~s}^{-1}$. The number density of the ISM was assumed to be equal to $0.04 \mathrm{~cm}^{-3}$, as predicted for $z \approx 0.5 \mathrm{kpc}$ by the model of vertical density profile of the Galactic disk by Dickey \& Lockman (1990; see their fig. 10). For these parameters the stand-off distance of the bow shock generated by the runaway system equals to $\approx 0.2 \mathrm{pc}$. At some point, the wind velocity was reduced by a factor of 20 to mimic slow outflow from the binary product, and, for the sake of simplicity, it was assumed that the wind momentum rate $\dot{M} v_{\infty}$ did not change. Correspondingly, the shape of the interface between the unperturbed and shocked ISM did not change as well, while the circumstellar wind material became a factor of 400 denser. After several thousand years (the time-scale of thermal adjustment), the wind parameters of the binary product were set to be equal to those of $\mathrm{CPD}-64^{\circ} 2731$, and the new, much faster, wind started to blow a bubble in the preceding slow wind material bounded in the upstream direction by the bow shock. In the simulations it was assumed that the stellar winds are spherically symmetric and that there is no instant (i.e. on a dynamical time-scale) mass loss (which is expected if CPD-64 2731 is a merger product).

Figure 8 shows synthetic maps of $22 \mu \mathrm{m}$ dust emission from the steady-state bow shock produced by a runaway star with wind parameters and space velocity identical to those of CPD- $64^{\circ} 2731$. Panels a)-d) show maps for four inclination angles of the line of sight to the symmetry axis of the simulation $(Y=0): 90^{\circ}, 60^{\circ}, 45^{\circ}$ and $33^{\circ}$. The latter angle corresponds to the orientation of the space velocity vector of $\mathrm{CPD}-64^{\circ} 2731$ derived from the proper motion and radial velocity measurements for this star. As expected, in all four projections the bow shock appears as an arc-like nebula with the associated star located close to the nose of the arc. This confirms that the nebula around CPD-64 2731 and the almost central location of this star in the nebula cannot be explained by the projection effect in the framework of the bow-shock model.

In Fig.9 we show evolution of the wind bubble blown by the fast wind from the binary product after its thermal adjustment. The panels plot synthetic maps of $22 \mu \mathrm{m}$ emission for the inclination angle of $33^{\circ}$. The shell around the bubble almost immediately appeared as an opened (arc-like) structure because of the presence of dense material ahead of the star (see panel a). At this time the nebula could be confused with a bow shock. After $\approx 1000$ yr of evolution the bubble expanded in the forward direction beyond the region occupied by the material of the preceding slow wind and started to interact with the unperturbed ISM. At this time the shell adopts a horseshoe shape (panel b). Later on (panel c) the shell became more rounded, while the maximum of its surface brightness has shifted downstream. At the same time, the star still remains close to the geometric centre of the bubble. At a later time (not shown in Fig.9), after the star will completely overrun the region occupied by the material of the slow wind, the bubble transformed into a bow shock identical to that shown in Fig. 8. except for a factor of $10^{1 / 2}$ larger stand-off distance due to a factor of 10 lower $n_{\mathrm{ISM}}$ adopted in the second simulation (cf. Meyer et al. 2014).

Although the model shell reproduces reasonably well the horseshoe shape of the nebula around CPD $-64^{\circ} 2731$, there is a notable distinction between them. Namely, the symmetry axis of the model nebula is parallel to the vector of stellar space velocity, while that of the observed nebula is misaligned by an angle of $\approx 30^{\circ}$ (see Fig. 6). A possible explanation of this misalignment is that the star is moving at an angle to a density gradient in the local medium. The density inhomogeneity could be inherent to the local ISM or caused by non-spherically symmetric density distribution in the slow wind, which is expected to be concentrated towards the orbital plane of the binary system, whose orientation could be arbitrary with respect to the vector of stellar velocity.

\section{CONCLUDING REMARKS}

Our interpretation of the nebula around CPD $-64^{\circ} 2731$ as a transient shell driven by the variable wind from the product 


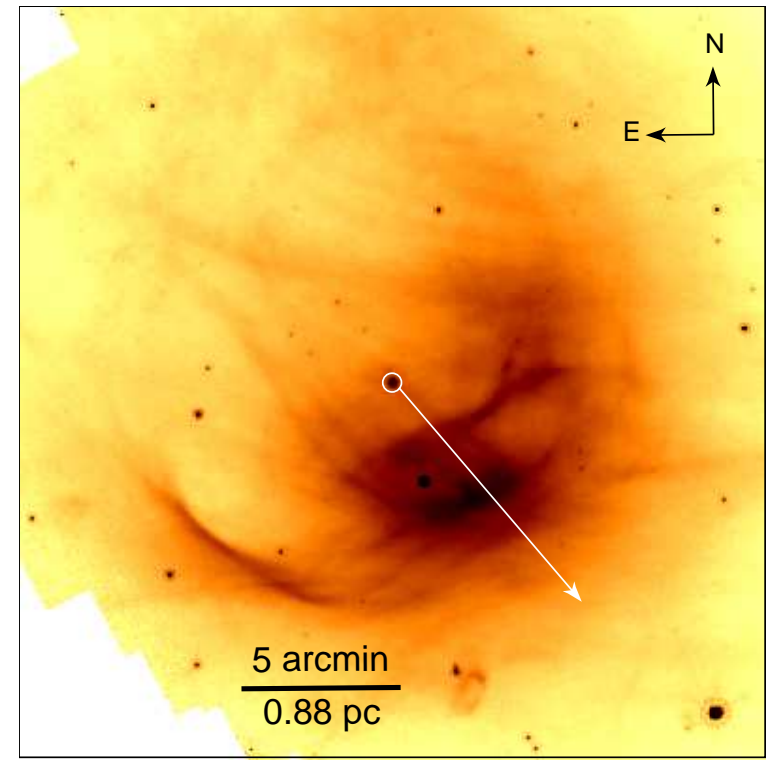

Figure 10. Spitzer $24 \mu \mathrm{m}$ image of a semi-circular nebula around the runaway star $\lambda$ Cep (marked by a circle). The arrow shows the direction of the peculiar transverse velocity of the star based on the Gaia DR2 parallax and proper motion measurements.

of binary interaction could also be applied to some other runaway stars whose nebulae do not look like bow shocks. For example, a semi-circular mid-infrared shell centred on the single (Gies 1987) runaway (Blaauw 1961) O6.5 I(n)fp (Sota et al. 2011) star $\lambda$ Cep (see Fig.10) might be a young bubble created by the variable mass loss from the binary merger product. This possibility is in line with the suggestion by Walborn et al. (2010) that the Onfp phenomenon could be caused by binary mass transfer or stellar mergers.

The idea that merger of massive binaries play an important role in evolution and observational manifestations of OB stars attracts more and more attention during the last decade. For example, it was suggested that binary merger could be responsible for the origin of strong magnetic field observed in some massive stars (e.g. Ferrario et al. 2009; Langer 2012). In particular, Schneider et al. (2016) proposed that two magnetic stars, HR 2949 (B3p IV) and $\tau$ Sco $(\mathrm{B} 0.2 \mathrm{~V})$, are the rejuvenated merger products, and noted that such objects could be distinguished from genuine single stars by the presence of circumstellar material ejected before and/or during the merger process. Interestingly, $\tau$ Sco is indeed surrounded by a curious infrared nebula (see Fig.11). The unevolved status of this star implies that the nebula might have been formed because of binary merger, although one cannot also exclude the possibility that it represents a radiation-pressure-driven bow wave (cf. van Buren \& McCray 1988; Ochsendorf et al. 2014).

Finally, we note that if $\mathrm{CPD}-64^{\circ} 2731$ is a merger product of two stars then it could posses a strong magnetic field. Thus, as noted above, spectropolarimetric observations of this star could be of high importance.

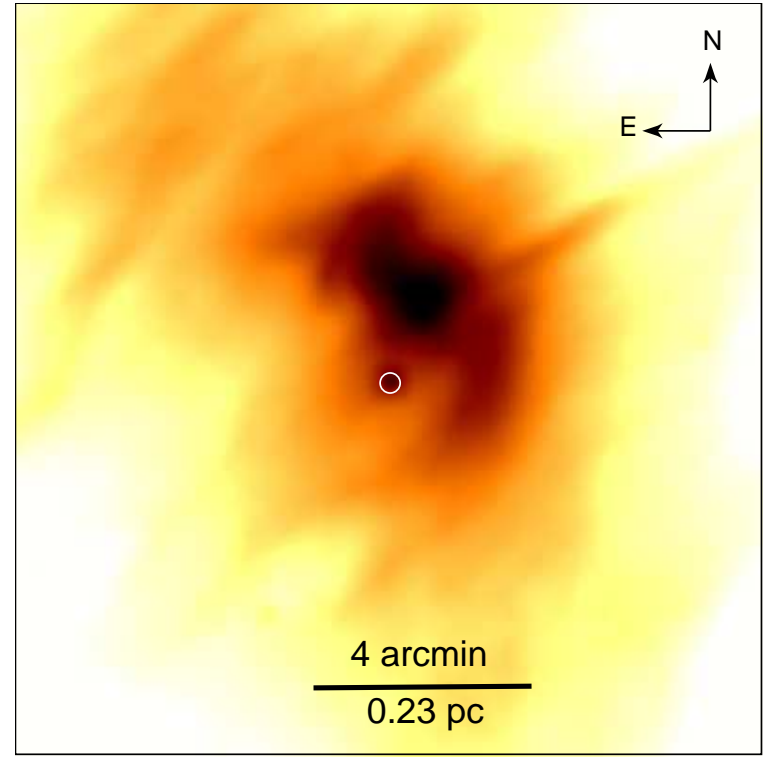

Figure 11. WISE $22 \mu \mathrm{m}$ image of a nebula around the magnetic star $\tau$ Sco (marked by a circle).

\section{SUMMARY}

We have discovered an almost complete (horseshoe-shaped) mid-infrared shell around the high-velocity $\left(\approx 160 \mathrm{~km} \mathrm{~s}^{-1}\right)$ runaway star $\mathrm{CPD}-64^{\circ} 2731$ with WISE. The shape of the shell and the nearly central location of $\mathrm{CPD}-64^{\circ} 2731$ within it argue against the interpretation of the shell as a bow shock. Spectral analyses of $\mathrm{CPD}-64^{\circ} 2731$, based on optical échelle spectroscopy with SALT, showed that we dial with a fast-rotating $\left(v \sin i \approx 300 \mathrm{~km} \mathrm{~s}^{-1}\right)$ main-sequence O5.5 star with a factor of 6-8 enhanced surface nitrogen abundance. The chemical abundances, high rotational velocity and large kinematic age of CPD-64 2731 exclude the possibility that this $\approx 40 \mathrm{M} \odot$ star was ejected from the Galactic plane because of dissolution of a binary system following supernova explosion. Instead, we have proposed that CPD $-64^{\circ} 2731$ was initially a compact binary system, which was dynamically ejected from a very massive $\left(\sim 10^{4} \mathrm{M} \odot\right)$ star cluster about $6 \mathrm{Myr}$ ago and recently experienced strong binary interaction (either a mass transfer or merger) leading to spin-up and rejuvenation of this star. This interaction has also led to a strong and slow wind, either from the fast rotating accretion star or bloated merger product, lasting for a Kelvin-Helmholtz time-scale (about several thousand years). After the thermal adjustment the binary product established a fast wind which started to blow a bubble within the material of the preceding slow wind, producing a transient short-lived ( $\sim 1000 \mathrm{yr}$ ) shell reminiscent that around $\mathrm{CPD}-64^{\circ} 2731$. This scenario was validated by means of $2 \mathrm{D}$ numerical modelling. We also have run a second simulation in which we modelled a bow shock produced by a runaway star whose wind parameters and space velocity were set to be identical to those of $\mathrm{CPD}-64^{\circ} 2731$. With this simulation we confirmed that the nebula around $\mathrm{CPD}-64^{\circ} 2731$ cannot be explained by the projection effect in the framework of bow-shock model. We also have proposed that the semi-circular nebula centred on the runaway Onfp star $\lambda$ Cep 
could be another case of a transient shell created because of changes in the mass-loss rate and wind velocity following the binary interaction (most likely a binary merger). Finally, we presented for the first time a WISE $22 \mu \mathrm{m}$ image of a curious nebula around the main-sequence magnetic star $\tau$ Sco, which is believed to be a rejuvenated binary merger product. This nebula could represent a circumstellar material ejected before and/or during the merger process or, alternatively, a radiation-pressure-driven bow wave.

\section{ACKNOWLEDGEMENTS}

We are grateful to I.D. Howarth (the referee) for useful comments and suggestions on the manuscript, and to J. Maíz Apellániz for useful discussion. Spectroscopic observations, data reduction and spectral modelling were supported by the Russian Foundation for Basic Research grant 16-02-00148. Interpretation of the observational data and numerical simulations were supported by the Russian Science Foundation grant No. 14-12-01096. AYK acknowledges support from the National Research Foundation (NRF) of South Africa. This work was supported in part by M.V.Lomonosov Moscow State University Program of Development and is based on observations collected with the Southern African Large Telescope (SALT), programmes 2017-1-MLT-005 and 2018-1-MLT-008. This work has made use of data products from the Wide-field Infrared Survey Explorer, which is a joint project of the University of California, Los Angeles, and the Jet Propulsion Laboratory/California Institute of Technology, funded by the National Aeronautics and Space Administration, the SIMBAD data base and the VizieR catalogue access tool, both operated at CDS, Strasbourg, France, the WEBDA data base, operated at the Department of Theoretical Physics and Astrophysics of the Masaryk University, and data from the European Space Agency (ESA) mission Gaia (https://www.cosmos.esa.int/gaia), processed by the Gaia Data Processing and Analysis Consortium (DPAC, https://www.cosmos.esa.int/web/gaia/dpac/consortium).

Funding for the DPAC has been provided by national institutions, in particular the institutions participating in the Gaia Multilateral Agreement.

\section{REFERENCES}

Bailer-Jones C. A. L., Rybizki J., Fouesneau M., Mantelet G., Andrae R., 2018, AJ, 156, 58

Barannikov A. A., 1993, Astron. Lett., 19, 420

Baranov V. B., Krasnobaev K. V., Kulikovskii A. G., 1970, Dokl. Akad. Nauk SSSR, 194, 41

Barnes S. I. et al., 2008, in McLean I. S., Casali M. M., eds, Proc. SPIE Conf. Ser. Vol. 7014, Ground-based and Airborne Instrumentation for Astronomy II. SPIE, Bellingham, p. $70140 \mathrm{~K}$

Blaauw A., 1961, Bull. Astron. Inst. Neth., 15, 265

Bramall D. G. et al., 2010, in McLean I. S., Ramsay S. K., Takami H., eds, Proc. SPIE Conf. Ser. Vol. 7735, Ground-based and Airborne Instrumentation for Astronomy III. SPIE, Bellingham, p. $77354 \mathrm{~F}$

Bramall D. G. et al., 2012, in McLean I. S., Ramsay S. K., Takami H., eds, Proc. SPIE Conf. Ser. Vol. 8446, Ground-based and
Airborne Instrumentation for Astronomy IV. SPIE, Bellingham, p. $84460 \mathrm{~A}$

Brighenti F., D'Ercole A., 1997, MNRAS, 285, 387

Brott I. et al., 2011, A\&A, 530, A115

Bruccato R. J., Mihalas D., 1971, MNRAS, 154, 491

Coelho P. R. T., 2014, MNRAS, 440, 1027

Cazorla C., Morel T., Nazé Y., Rauw G., Semaan T., Daflon S., Oey M. S., 2017, A\&A, 603, A56

Chakravarthy S., Osher S., 1985, AIAA Pap., N 85-0363

Chini R., Hoffmeister V. H., Nasseri A., Stahl O., Zinnecker H., 2012, MNRAS, 424, 1925

Conti P. S., Alschuler W. R., 1971, ApJ, 170, 325

Cordes J. M., Lazio T. J. W., 2002, preprint (astro-ph/0207156

Cowie L. L., McKee C. F., Ostriker J. P., 1981, ApJ, 247, 908

Crause L. A. et al., 2014, in Ramsay S. K., McLean I. S., Takami H., eds, Proc. SPIE Conf. Ser. Vol. 9147, Ground-based and Airborne Instrumentation for Astronomy V. SPIE, Bellingham, p. $91476 \mathrm{~T}$

Crawford S. M. et al., 2010, in Silva D. R., Peck A. B., Soifer B. T., eds, Proc. SPIE Conf. Ser. Vol. 7737, Observatory Operations: Strategies, Processes, and Systems III. SPIE, Bellingham, p. 773725

Cutri R. M. et al., 2003, VizieR Online Data Catalog, 2246

Cutri R. M. et al., 2014, VizieR Online Data Catalog, 2328

Decin L., Hony S., de Koter A., Justtanont K., Tielens A. G. G. M., Waters L. B. F. M., 2006, A\&A, 456, 549

de Mink S. E., Langer N., Izzard R. G., Sana H., de Koter A., 2013, ApJ, 764, 166

Dickey J. M., Lockman F. J., 1990, ARA\&A, 28, 215

Eldridge J. J., Langer N., Tout C. A., 2011, MNRAS, 414, 3501

Ferrario L., Pringle J. E., Tout C. A., Wickramasinghe D. T., 2009, MNRAS, 400, L71

Ferrero L., Le Du P., Mulato L., Outters N., Zoll S., 2015, L'astronomie, 129, 42

Fossati L. et al., 2016, A\&A, 592, A84

Gaia Collaboration Brown A. G. A., Vallenari A., Prusti T., de Bruijne J. H. J., Babusiaux C., Bailer-Jones C. A. L., 2018, A\&A, 616, A1

García-Segura G., Mac Low M.-M., Langer N., 1996a, A\&A, 305, 229

García-Segura G., Langer N., Mac Low M.-M., 1996b, A\&A, 316, 133

Gies D. R., 1987, ApJS, 64, 545

Glebbeek E., Gaburov E., Portegies Zwart S., Pols O. R., 2013, MNRAS, 434, 3497

Godunov S. K., Zabrodine A. V., Ivanov M. Y., Kraiko A. N., Prokopov G. P., 1979, Résolution Numérique des Problèmes Multidimensionnels de la Dynamique des Gaz, Editions MIR, Moscow

Götberg Y., de Mink, S. E., Groh J. H., 2017, A\&A, 608, A11

Gvaramadze V. V., Gualandris A., 2011, MNRAS, 410, 304

Gvaramadze V. V., Kniazev A. Y., 2017, in Miroshnichenko A.S., Zharikov S.V., Korcakova D., Wolf M., eds., ASP Conf. Ser. Vol. 508, The B[e] Phenomenon. Forty Years of Studies. Astron. Soc. Pac., San Francisco, p. 207

Gvaramadze V. V., Kniazev A. Y., Fabrika S., 2010, MNRAS, 405, 1047

Gvaramadze V. V., Kniazev A. Y., Kroupa P., Oh S., 2011, A\&A, 535, A29

Gvaramadze V. V. et al., 2012a, MNRAS, 421, 3325

Gvaramadze V. V., Weidner C., Kroupa P., Pflamm-Altenburg J., 2012b, MNRAS, 424, 3037

Henden A. A, Templeton M., Smith T. C., Levine S., Welch D., 2016, VizieR Online Data Catalog, 2336, 0

Hillier D. J., Miller D. L., 1998, ApJ, 496, 407

Hillier D. J., Miller D. L., 1999, ApJ, 519, 354

Hubeny I., Lanz T., 1995, ApJ, 439, 875

Izmodenov V. V., Alexashov D. B., 2015, ApJS, 220, 32 
Kniazev A. Y., Gvaramadze V. V., Berdnikov L. N., 2016, MNRAS, 459, 3068

Kroupa P., 1998, MNRAS, 298, 231

Kudritzki R.-P., Puls J., 2000, ARA\&A, 38, 613

Lada C. J., Lada E. A., 2003, ARA\&A, 41, 57

Langer N., 1998, A\&A, 329, 551

Langer N., 2012, ARA\&A, 50, 107

Lanz T., Hubeny I., 2003, ApJS, 146, 417

Leonard P. J. T., Duncan M. J., 1990, AJ, 99, 608

Lynga G., 1969, Arkiv för Astronomii, 5, 181

Maíz Apellániz J. et al., 2016, ApJS, 224, 4

Martins F., Hillier D. J., 2012, A\&A, 545, A95

Martins F., Plez B., 2006, A\&A, 457, 637

Martins F., Schaerer D., Hillier D. J., 2005, A\&A, 436, 1049

Mason B. D., Hartkopf W. I., Gies D. R., Henry T. J., Helsel J. W., 2009, AJ, 137, 3358

Massey P., Puls J., Pauldrach A. W. A., Bresolin F., Kudritzki R. P., Simon T., 2005, ApJ, 627, 477

McLean B. J., Greene G. R., Lattanzi M. G., Pirenne B., 2000, in Manset N., Veillet C., Crabtree D., eds, ASP Conf. Ser. Vol. 216, Astronomical Data Analysis Software and Systems IX. Astron. Soc. Pac., San Francisco, p. 145

Mermilliod J. C., 1995, in Egret D., Albrecht M., eds, Information and Online Data in Astronomy. Kluwer, Dordrecht, p. 127

Meyer D. M.-A., Mackey J., Langer N., Gvaramadze V. V., Mignone A., Izzard R. G., Kaper L., 2014, MNRAS, 444, 2754

Moultaka J., Ilovaisky S. A., Prugniel P., Soubiran C., 2004, PASP, 116, 693

Myasnikov A., 1997, Preprint No. 585, Institute for Problems in Mechanics, Russian Academy of Sciences

Nieva M.-F., Przybilla N., 2012, A\&A, 539, A143

Ochsendorf B. B., Cox N. L. J., Krijt S., Salgado F., Berné O., Bernard J. P., Kaper L., Tielens A. G. G. M., 2014, A\&A, $563, \mathrm{~A} 65$

Oh S., Kroupa P., 2012, MNRAS, 424, 65

Oh S., Kroupa P., 2016, A\&A, 590, A107

Parker Q. A. et al., 2005, MNRAS, 362, 689

Pauldrach A. W. A., Puls J., 1990, A\&A, 237, 409

Petrovic J., Langer N., van der Hucht K. A., 2005, A\&A, 435, 1013

Portegies Zwart S. F., 2000, ApJ, 544, 437

Poveda A., Ruiz J., Allen C., 1967, Bol. Obser. Tonantzintla Tacubaya, 4,86

Ramírez-Agudelo O. H. et al., 2013, A\&A, 560, A29

Reed B. C., 2000, AJ, 120, 314

Reid M. J., Menten K. M., Zheng X. W., Brunthaler A., Xu Y., 2009, ApJ, 705, 1548

Repolust T., Puls J., Herrero A., 2004, A\&A, 415, 349

Sana H. et al., 2012, Science, 337, 444

Schneider F. R. N., Izzard R. G., Langer N., de Mink S. E., 2015, ApJ, 805, 20

Schneider F. R. N., Podsiadlowski P., Langer N., Castro N., Fossati L., 2016, MNRAS, 457, 2355

Schlafly E. F., Finkbeiner D. P., 2011, ApJ 737, 103

Schönrich R., Binney J., Dehnen W., 2010, MNRAS, 403, 1829

Simón-Díaz S., Herrero A., 2007, A\&A, 468, 1063

Simón-Díaz S., Herrero A., 2014, A\&A, 562, A135

Skrutskie M. F. et al., 2006, AJ, 131, 1163

Sota A., Maíz-Apellániz J., Walborn N.R., Alfaro E.J., Barbá R.H., Morrell N.I., Gamen R.C., Arias J.I., 2011, ApJS, 139, 24

Sota A., Maíz-Apellániz J., Morrell N. I., Barbá R. H., Walborn N. R., Gamen R. C., Arias J. I., Alfaro E. J., 2014, ApJS, 211,10

Stone R. C., 1979, ApJ, 232, 520

Tutukov A. V., 1978, A\&A, 70, 57

van Buren D., McCray R., 1988, ApJ, 329, L93

van Oijen J. G. J., 1989, A\&A, 217, 115
Vijapurkar J., Drilling J. S., 1993, ApJS, 89, 293

Vink J. S., 2018, preprint (arXiv:1808.06612)

Walborn N. R. et al., 2010, AJ, 139, 1283

Weidner C., Vink J. S., 2010, A\&A, 524, A98

Wellstein S., Langer N., Braun H., 2001, A\&A, 369, 939

Wright E. L. et al., 2010, AJ, 140, 1868 